\title{
Efficiency and Distortions in a Production Economy with Heterogeneous Beliefs Working Paper*
}

\author{
Christian Heyerdahl-Larsen ${ }^{\dagger} \quad$ Johan Walden ${ }^{\ddagger}$
}

December 12, 2014

\begin{abstract}
In a production economy, in which agents have heterogeneous beliefs and a social planner has incomplete knowledge about which beliefs are correct, we introduce the concept of Incomplete Knowledge (IK) efficiency. IK-inefficient allocations can be improved upon without taking a stand on which belief, among a whole set of reasonable beliefs, is correct. We show that competitive equilibrium under heterogeneous beliefs is always IK-inefficient, and decompose this inefficiency into investment and speculative distortions. Overinvestment occurs in economies in which agents' elasticity of intertemporal substitution is high, whereas underinvestment arises when their elasticity is low. Using the IK concept to define mispricing, we show that equilibrium mispricing never arises intratermporally, but that intertemporal mispricing is generically present in economies with rich state spaces and heterogeneous beliefs. Finally, we argue that investment distortions may be easier to address by a social planner than speculative distortions in economies with idiosyncratic endowment shocks, and also show that transaction taxes may be welfare decreasing in such economies.
\end{abstract}

Keywords: Speculation, heterogeneous beliefs, mispricing, investments, welfare.

\footnotetext{
${ }^{*}$ Comments are welcome. We thank participants at the Berkeley Finance lunch seminar, Bernard Dumas, Willie Fuchs and Brett Green for helpful comments and suggestions. Walden thanks INSEAD and NYU Stern for hosting visits during which part of this research was carried out.

${ }^{\dagger}$ London Business School, Regent's Park, London, NW1 4SA, United Kingdom. Email: cheyerdahllarsen@london.edu

${ }^{\ddagger}$ Haas School of Business, University of California at Berkeley, 545 Student Services Building \#1900, CA 947201900. E-mail: walden@haas.berkeley.edu, Phone: +1-510-643-0547. Fax: +1-510-643-1420.
} 


\section{Introduction}

In the aftermath of the financial crisis, welfare analysis in economies with heterogeneous beliefs has received renewed attention in several recent studies (Brunnermeier et al. 2013; Gilboa et al. 2013; Gayer et al. 2013; Blume et al. 2014). These studies suggest that speculative trades between agents with different beliefs, although increasing the ex ante expected utility of each agent, is costly from an ex post perspective, since some agents must necessarily be wrong and will end up very poor after speculating away much of their wealth. Speculation in the heterogeneous beliefs economy, instead of creating welfare by allowing agents to hedge risks and smooth consumption across states, generates volatility of consumption and wealth at the individual level (see Yan 2008, and Fedyk, Heyerdahl-Larsen, and Walden 2013). Speculation also affects asset price dynamics, increasing trading volume and price volatility, and potentially leading to mispricing (see Kubler and Schmedders 2012, and Simsek 2013 for recent contributions), effects that may be viewed as negative in their own right.

The potential policy implications with such an ex post measure of welfare are, of course, huge, suggesting that moving away from the friction-free complete market equilibrium - even when it is implementable - may actually be welfare improving. It is therefore vital to understand when such welfare improvements are possible, how speculative distortions can be identified, and what type of actions the social planner may take to mitigate them. In this paper, we study these questions.

We introduce a two-date economy with production and idiosyncratic shocks to agents' endowments, to understand how equilibrium is affected by heterogeneous beliefs. The state space is perfectly general. Specifically, we are interested in what can be said about equilibrium outcomes without taking a strong stand on what are the correct beliefs in the economy, based on the view that requiring a social planner to identify correct beliefs in order to take action is to set the bar too high. Indeed, a crucial role of markets is to allow for information dissemination and aggregation, beyond the information available to any individual agent, and it is thus unclear how the social planner would obtain such superior beliefs. We therefore focus on properties of equilibrium that hold for any belief within a set of reasonable beliefs. We introduce the concept of a welfare measure that is Ex Post efficient under Incomplete Knowledge about true probabilities, i.e., an EPIK welfare measure. A Social Planner who uses the EPIK measure is then a SPEPIK.

We are interested in what can be said in general about the equilibrium outcome in the heterogeneous beliefs economy, from the SPEPIK's perspective. Our analysis has similarities with, and builds upon, concepts introduced in Gilboa et al. (2013), Brunnermeier et al. (2013) and Gayer et al. (2013), but also has significant differences. Crucial components of our analysis are that (i) the SPEPIK does not know the correct probability distribution, (ii) the market has a role of allocating productive resources (production economy), and (iii) that agents face idiosyncratic endowment shocks.

Without production and with no idiosyncratic shocks, the only role of the market is speculative, and it is straightforward for a social planner to determine which allocations are ex post efficient. The situation is not as clear in the general economy with production and idiosyncratic endowment 
shocks, in which the social planner has incomplete knowledge about true probability distributions. First, markets for contingent claims on idiosyncratic risk now have the dual roles of allowing for hedging of endowment shocks and of allowing for speculation. The former role is welfare increasing, the latter is not. Second, belief heterogeneity may have real effects in a production economy by affecting investments. Again, the implication for the social planner is a priori unclear. Third, whereas asset mispricing is straightforward to identify when true probability distributions are known, it is less clear if and when mispricing can arise when they are not.

We introduce the concept of Incomplete Knowledge (IK) efficiency. Loosely speaking, an allocation, $a$, is IK-inefficient if there is an alternative allocation that dominates $a$, for some reasonable beliefs and is not dominated by $a$ for any reasonable beliefs. We show that any competitive equilibrium with heterogeneous beliefs is IK-inefficient. The introduction and equilibrium analysis of IK-efficiency constitutes our first contribution.

Our second contributions is to show that when agents agree on aggregate risk, disagreement about idiosyncratic risk leads to real distortions that can be identified by a SPEPIK. The direction of the distortion is closely related to agents' elasticity of intertemporal substitution (EIS). When the EIS is less than one, disagreement unambiguously leads to underinvestment, whereas when the EIS is greater than one, disagreement generates overinvestment. The intuition behind the result is straightforward. Disagreement and trading, together generate a speculative wealth effect, where all agents believe that they are better off in the economy with disagreement than in one without it, because of expected gains from speculation. They therefore readjust their consumption and investment based on these expectations of higher future wealth.

Third, we study potential policy implications. Although the source of inefficiency is the speculation generated by heterogeneous beliefs, we argue that mitigating such speculation by shutting down markets may be costly when agents are exposed to idiosyncratic endowment shocks. Alternative approaches may be to tax transactions (a Tobin tax), and taxes or subsidies on investments. Within our setting, it is shown that transaction taxes may be welfare decreasing, whereas investment taxes or subsidies can always be chosen so that their effect is welfare improving.

As a fourth contribution, we study equilibrium mispricing. We discuss two types of distortions: Intertemporal mispricing arises when prices of contingent claims on future states, expressed in terms of current consumption, cannot be justified by any reasonable beliefs. Intratemporal mispricing, on the other hand, is defined by comparing the relative prices of two assets that make payments at the same point in time. We show that intratemporal mispricing never arises in equilibrium, and thus cannot be used to detect distortions. Intertemporal mispricing, on the other hand, generically arises as long as the state space and belief heterogeneity is sufficiently rich. A key takeaway from our analysis is that mispricing is easiest to identify in assets for which there is little belief heterogeneity, since the range of reasonable prices for such assets is small.

The rest of the paper is organized as follows: In the next section, we discuss welfare under heterogeneous beliefs, connect our approach to previous literature, and provide a motivation for using the incomplete knowledge welfare measure. In Section 3, we introduce and analyze the general model. Section 4 provides results on speculative, investment and price distortions and Section 5 
covers potential policy implications. Section 6 discusses extensions and Section 7 concludes. All proofs are delegated to an Appendix.

\section{Efficiency under heterogeneous beliefs}

The EPIK welfare measure has two components: The first is the use of an ex post measure. An ex post welfare measure identifies speculation and the individual consumption volatility it is associated with as inefficient, in contrast to an ex ante measure, under which the speculation that may arise in a complete Walrasian market with heterogeneous beliefs is viewed as efficient. An argument against the ex ante measure is that all agents agree that they cannot all be correct in their beliefs, and it can therefore be argued that these welfare gains are spurious. With an ex post view, for an allocation to be viewed as efficient it must be efficient under some "true" probability distribution. Although agents may not agree about which probability distribution is "true," they all agree that such a "true" distribution exists and - the argument goes - they should consequently agree that allocations that are inferior under any such true distribution are inefficient. ${ }^{1}$ It has also been argued that policies that are based on ex post measures, if they restrict the actions of agents, could be viewed as paternalistic (see, e.g., Harris and Olewiler (1979), and Fleurbaey (2010)). For further discussion of the use of an ex post measure, which we take as given, we refer to the extensive previous literature, see Starr (1973), Harris and Olewiler (1979), Hammond (1981), Harris (1978), Portney (1992), Hausman and McPherson (1994), Pollak (1998), Salanie and Treich (2009), and also to recent studies by Gilboa et al. (2013), Brunnermeier et al. (2013), Gayer et al. (2013), and Blume et al. (2014).

The second component of the EPIK welfare measure is based on a sober view of the social planner's ability to identify the "true" probability distribution. Such a view may be considered overly pessimistic, but we note that there are plenty of historical examples when it would have been appropriate. For example, it is known that the presence of overoptimistic investors in the market together with short-sale constraints may give rise to price and investment bubbles (e.g., Scheinkman and Xiong 2003 and Gilchrist et al. 2005). However, although bubbles and overoptimism may be easy to identify in hindsight, there is often considerable ex ante uncertainty about their presence.

There was no consensus, and relatively few warnings about there being a bubble in the run-up years before the crash of the U.S. housing market in 2007, for example. Even former Chairman of the Federal Reserve Board, Alan Greenspan, admitted to not "getting" that there was trouble on the horizon until right before the crash. ${ }^{2}$ During the New Economy boom and the associated dot-com bubble, between 1997-2000, warnings were issued by some, whereas others argued that discontinuous technological transition made "Old Economy" valuation formulas out-of-date. Shiller (2000) warned about a bubble generated by "irrational exuberance," borrowing the term from

\footnotetext{
${ }^{1}$ We note that the expected utility decomposition of agent preferences into a utility part and a probabilistic part is interpreted literally in this argument. Without such a literal interpretation, e.g., if the expected utility specification is viewed as merely a way of representing preferences that are linear over probabilities, in line with the axiomatic approach in Savage (1954), the argument for using the ex post expected utility measure is not as straightforward.

${ }^{2}$ CBS 60 Minutes, interview, September 13, 2007.
} 
Alan Greenspan. Pastor and Veronesi (2006), in contrast, argue that the uncertainty about future growth rates in the late 1990's may well have justified the valuation of the NASDAQ. Moreover, there are many examples throughout history of technological innovations that turned out to be transformative, but that were originally dismissed by many as fads, including the automobile, computers, mobile phones and the Internet. A key question is then if and how distortions and mispricing can be identified without exact knowledge about which beliefs are correct.

In line with the sober view of the social planner's ability to identify correct beliefs, in the baseline version of our model we assume that no individual agent's beliefs can be ruled out as being incorrect. Specifically, we will consider a set of reasonable beliefs that is rich enough to contain all individual agent's beliefs. Our approach is general, however, and can easily be adapted to situations where the social planner decides to use a different set of reasonable beliefs, e.g., excluding those of some agents, that are obviously wrong.

\section{$2.1 \quad$ Notation}

Vectors are enumerated by subscripts, $p=\left(p_{1}, p_{2}, \ldots, p_{M}\right)$, and also by superscripts, $k=\left(k^{1}, k^{2}, \ldots, k^{N}\right)$, to conveniently separate vectors over states from vectors over agents. We use a tilde above a variable if we wish to stress that it is a random variable (e.g., $\tilde{c}$ ), but avoid this somewhat cumbersome notation when possible.

The $M$-dimensional open unit simplex is

$$
S^{M}=\left\{x \in \mathbb{R}^{M}: x_{n}>0, \sum_{n=1}^{n} x_{n}=1\right\},
$$

and its closure is $\bar{S}^{M}$. The convex hull of a set $\Lambda \subset \mathbb{R}^{M}$ is denoted by

$$
C H(\Lambda)=\left\{\sum_{m=1}^{M+1} x_{m} \lambda_{m}: x \in \bar{S}^{M+1}, \lambda_{m} \in \Lambda, m=1, \ldots M+1\right\}
$$

The image of a set, $A$, under the function $f$ is $f(A)=\{f(a): a \in A\}$, and the preimage is $f^{-1}(A)=\{a: f(a) \in A\}$. The power set of the set $\Omega, 2^{\Omega}$, is defined as the set of all subsets of $\Omega$ : $2^{\Omega}=\{P: P \subset \Omega\}$. The set difference between sets $A$ and $B$ is $A \backslash B$, and the complement of $A$ in a universal set, $U$, is $A^{c}=U \backslash A$. We use $\neg$ for logical negation, $\neg A$ is "not $A$."

\subsection{Efficiency}

To justify the IK-efficiency measure, we introduce a simple example with two risk averse expected utility maximizing agents, three mutually exclusive production technologies, and two states. The expected utility of agent $n \in\{1,2\}$ is

$$
U^{n}=q \sqrt{c_{1}^{n}}+(1-q) \sqrt{c_{2}^{n}}
$$


where $c_{m}^{n}$ is the consumption of agent $n$ in state $m \in\{1,2\}$, and $q$ is the probability for state 1 to occur. The first production technology is risk free and generates a total output of 2 units of the consumption good in either state. The second technology is risky, generating 18 units in state 1 and 0 units in state 2 , as is the third technology which generates 18 units in state 2 and 0 in state 1.

We consider 4 allocations, captured by the set $\mathcal{A}=\left\{a_{1}, a_{2}, a_{3}, a_{4}\right\}$. In allocations $a_{1}-a_{3}$, the consumption good is split equally between the two agents, after choosing each of the three production technologies. Allocation $a_{4}$ allows for speculation. Specifically, we assume that the two agents disagree about the probability for state 1 to occur, $q$. Agent 1 believes that the probability is $q^{1}=0.9$, whereas agent 2 believes that it is $q^{2}=0.1$. The SPEPIK, not knowing which beliefs are correct, views any probability in the interval $\mathcal{Q}_{R}=[0.1,0.9]$ as reasonable. Here, the subscript, $R$, represents that this is a set of "Reasonable" probabilities.

In allocation $a_{4}$, the risk-free production technology is used, as in allocation $a_{1}$, but agent 1 receives all the output in state 1 , whereas agent 2 receives all the output in state 2 . The consumption by the two agents for different allocations and states is shown in Table 1. We focus on these four allocations, but would more generally expand the set of feasible allocations to allows for arbitrary redistribution of the consumption good between the two agents in each state.

\begin{tabular}{|c|c|c|c|c|c|c|c|c|}
\hline Allocation, & \multicolumn{2}{|c|}{$a_{1}$} & \multicolumn{2}{|c|}{$a_{2}$} & \multicolumn{2}{|c|}{$a_{3}$} & \multicolumn{2}{|c|}{$a_{4}$} \\
\hline Agent & 1 & 2 & 1 & 2 & 1 & 2 & 1 & 2 \\
\hline State 1 & $\overline{1}$ & 1 & 9 & 9 & 0 & $\overline{0}$ & $\overline{2}$ & $\overline{0}$ \\
\hline State 2 & 1 & 1 & 0 & 0 & 9 & 9 & 0 & 2 \\
\hline
\end{tabular}

Table 1: Four allocations in economy with two agents and two states. Allocation $a_{1}$ and $a_{4}$ are based on investments in the risk-free technology, with equal $\left(a_{1}\right)$ and unequal $\left(a_{4}\right)$ sharing between agents in the two states. Allocation $a_{3}$ and $a_{4}$ both have equal sharing, but invest in risky technologies that pay off in state 1 and 2, respectively.

It is easy to verify that with an ex ante welfare measure, the only inefficient allocation is $a_{1}$, which both agents agree is dominated by $a_{4}$, based on their different beliefs. But both agents also agree that the welfare improvement is purely speculative, and that whatever the true $q$ is, any allocation in which individual consumption shares vary across states can be improved upon by risk sharing. According to the ex post view, $a_{4}$ is therefore inefficient.

How should the SPEPIK determine which allocations are efficient, given an ex post measure where any probability $q \in \mathcal{Q}_{R}$ is viewed as reasonable? Figure 1 shows the agents' expected utilities as a function of $q$. In the left panel, allocations $a_{1}-a_{3}$ are compared. Since the agents share the total output equally, their expected utilities are the same for any given allocation and $q$. We see that allocation $a_{3}$ dominates the others for low $q$, but is inferior for high $q$. Similarly, allocation $a_{2}$ dominates the others for high $q$ but is inferior for low $q$. Thus, both $a_{2}$ or $a_{3}$ are efficient for some beliefs. We argue that a SPEPIK, who does not know $q$, should therefore also view $a_{1}$ as efficient, 
since although this allocation is dominated for any specific $q$ by some other allocation, without further knowledge about $q$ it is impossible for the SPEPIK to choose which other allocation to replace $a_{1}$ with.
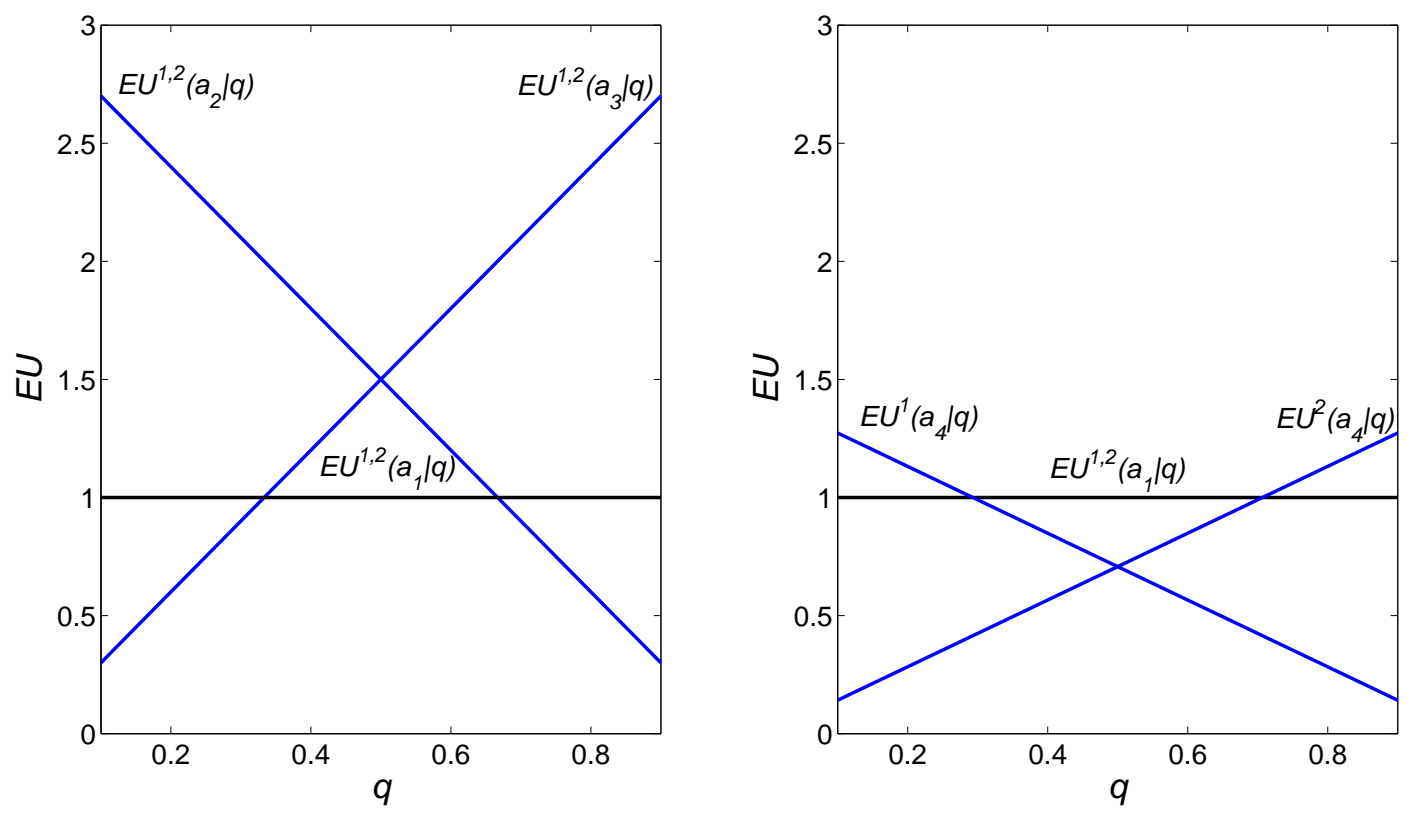

Figure 1: Expected utilities of agents as a function of $q$. Left panel: Comparing allocations $a_{1}$, $a_{2}$ and $a_{3}$. Right panel: Comparing allocations $a_{1}$ and $a_{4}$.

The right panel in Figure 1 compares allocation $a_{1}$ with $a_{4}$. The horizontal (black) line represents the (same) utility of the two agents under the risk free allocation $a_{1}$, whereas the sloped (blue) lines represent the utilities of the two agents under the risky allocation $a_{4}$. For low and high $q \mathrm{~s}$, one of the agents is better off under $a_{4}$ than under $a_{1}$, whereas the other is worse off, and for $q$ close to 0.5 both are worse off. Now, the reason one agent is better off for extreme $q$ 's is exactly because of the type of speculative redistributions that the ex post measure does not recognize as welfare improving. Regardless of $q$, allocation $a_{4}$ is therefore inferior from an ex post perspective. What changes with $q$ under allocation $a_{4}$ is which one of the two agents, who has the speculative advantage. A SPEPIK with well-defined Pareto weights can therefore always improve upon $a_{4}$, regardless of what those Pareto weights are. So, to summarize, in this example it is reasonable to require the SPEPIK to treat allocations $a_{1}-a_{3}$ as efficient, but $a_{4}$ as inefficient.

To formalize the intuition above, we introduce the Benthamite welfare function, $\mathcal{U}$, given homogeneous beliefs, $q$, and Pareto weights, $\lambda \in S^{N}$,

$$
\mathcal{U}(a \mid q, \lambda)=\sum_{n} \lambda^{n} U^{n}(a \mid q)
$$

where $U^{n}(a \mid q)$ in our specific example is defined in (1), and more generally is a finite-valued expected 
utility function. Note that the welfare function uses the same $q$ for all agents, being an ex post measure. As a precursor to our analysis in subsequent sections, we define this and the concepts to follow in full generality for $N \geq 2$ agents and a general set of feasible allocations, $\mathcal{A}$.

If $\mathcal{U}(b \mid q, \lambda)>\mathcal{U}(a \mid q, \lambda)$ for two allocations, $a, b \in \mathcal{A}$, we write $b \succ_{q}^{\lambda} a$. It follows easily that the standard definition of Pareto efficiency under homogeneous beliefs can be formulated as

\section{Definition 1 (Pareto dominance and efficiency with homogeneous beliefs)}

(i) Allocation $b$ dominates a given $q, b \succ_{q} a$, if $b \succ_{q}^{\lambda} a$ for all $\lambda \in S^{N}$.

(ii) Allocation $b$ is not dominated by a given $q, a \succeq_{q} b$, if $\neg\left(b \succ_{q} a\right)$.

(iii) Allocation $b$ is efficient given $q, b \in E_{q}$, if $\forall a \in \mathcal{A}: b \succeq_{q} a$.

Moving onto the heterogeneous beliefs economy, we introduce the concept of IK-dominance with respect to specific Pareto weights, which we then use to define Incomplete Knowledge (IK) efficiency:

Definition 2 Allocation $b$

(i) IK-dominates $a$ with respect to Pareto weights $\lambda, b \succ^{\lambda} a$, if:

$$
\left(\forall q \in \mathcal{Q}_{R}: b \succeq_{q}^{\lambda} a\right) \text { and }\left(\exists q \in \mathcal{Q}_{R}: b \succ_{q}^{\lambda} a\right),
$$

(ii) is not IK-dominated by $a$ with respect to Pareto weights $\lambda, a \succeq^{\lambda} b$, if $\neg\left(b \succ^{\lambda} a\right)$, i.e., if

$$
\left(\exists q \in \mathcal{Q}_{R}: a \succ_{q}^{\lambda} b\right) \quad \text { or }\left(\forall q \in \mathcal{Q}_{R}: a \succeq_{q}^{\lambda} b\right)
$$

\section{Definition 3 (IK-efficiency) Allocation a is}

(i) IK-efficient, $a \in E^{I K}$, if $\exists \lambda \in \bar{S}^{N}, \forall b \in \mathcal{A}, a \succeq^{\lambda} b$.

(ii) IK-inefficient, if $a \in\left(E^{I K}\right)^{c}$, i.e., if $\forall \lambda \in \bar{S}^{N}, \exists b \in \mathcal{A}: b \succ^{\lambda} a$.

An IK-inefficient allocation is thus one for which whatever the Pareto weights in the SPEPIK's welfare function, there exists another allocation that is not dominated by the first regardless of which reasonable $q$ is correct, and that dominates the first for some reasonable $q$. Thus, an IK-inefficient allocation is operational for the SPEPIK, who can move to an allocation $b$ that is consistent with the planner's Pareto weights, without taking a stand on $q$. The concept thus requires the social planner to have a well-defined $\lambda$, but requires no knowledge about $q \in \mathcal{Q}_{R}$.

We also introduce two convenient IK dominance concepts:

Definition 4 (IK-dominance) Allocation $b$ is said to

(i) weakly IK-dominate allocation $a$, if $\exists \lambda \in \bar{S}^{N}: b \succ^{\lambda} a$. 
(ii) strongly IK-dominate allocation $b$, if $\forall \lambda \in \bar{S}^{N}, b \succ^{\lambda} a$.

It follows immediately that a necessary condition for an allocation to be IK-efficient is that it is not strongly IK-dominated by any other allocation, and that a sufficient condition is that it is not weakly IK-dominated by any other allocations.

IK-dominance formalizes the intuition we described in the previous example. Indeed, it is straightforward to verify that allocations $a_{1}-a_{3}$ in the example are IK-efficient, whereas $a_{4}$ is IK-inefficient.

\subsection{Comparison with other efficiency measures}

We compare the IK-efficiency concept with two other concepts, namely belief neutral efficiency and so-called U-efficiency.

The concepts of belief neutral efficiency and inefficiency are introduced in Brunnermeier et al. (2013). With their definition, some allocations are neither classified as efficient, nor inefficient. To avoid this failure of principum tertii exclusi and the associated cumbersome terminology of "not belief neutrally inefficient allocations," we use the term weakly belief neutrally efficient for an allocation that is not belief neutrally inefficient.

\section{Definition 5 Allocation a is}

(v) Belief Neutrally efficient, $a \in E^{B N}$, if $\forall q \in \mathcal{Q}_{R}, \forall b \in \mathcal{A}: a \succeq_{q} b$,

(vi) Weakly Belief Neutrally efficient, $a \in \bar{E}^{B N}$, if $\exists q \in \mathcal{Q}_{R}, \forall b \in \mathcal{A}: a \succeq_{q} b$,

(vii) Belief Neutrally inefficient if $a \in\left(\bar{E}^{B N}\right)^{c}$, i.e., if $\forall q \in \mathcal{Q}_{R}, \exists b \in \mathcal{A}: b \succ_{q} a$.

We note that to be able to act on a belief neutrally inefficient allocation, the social planner in general needs to know the true probability distribution, contrary to our SPEPIK assumptions, since the allocation that dominates a belief neutrally inefficient allocation is allowed to depend on $q$. We also note that belief neutral efficiency is a strong requirement, since it requires a single allocation to be efficient for all reasonable $q$ 's.

In our example, $a_{1}$ is belief neutrally inefficient, since for any $q$ at least one of $a_{2}$ and $a_{3}$ dominates $a_{1}$, as seen in the left panel of Figure 1. Moreover, no allocation is belief neutrally efficient, since any allocation is dominated by another for some $q$. As a consequence, neither of the two concepts is operational for a SPEPIK in this example.

We next introduce the concept of U-efficiency, which is closely related to Unanimity Pareto dominance introduced in Gayer et al. (2013):

\section{Definition 6 Allocation a is}

(iii) U-efficient, $a \in E^{U}$, if $\forall b \in \mathcal{A}, \exists q \in \mathcal{Q}_{R}: a \succeq_{q} b$, 
(iv) U-inefficient if $a \in\left(E^{U}\right)^{c}$, i.e., if $\exists b \in \mathcal{A}, \forall q \in \mathcal{Q}_{R}: b \succ_{q} a$.

The requirement in Gayer et al. (2013) for an allocation to be Unanimity Pareto dominated by another is stronger than our requirement for the allocation to be U-inefficient, in that they require every agent who participates in the transaction leading to the new allocation to be strictly better off, whereas we only require one agent to be strictly better off and the rest to be weakly better off. Therefore, an allocation that is U-efficient under our definition is not Unanimity Pareto dominated by any other allocation, using their definition.

We note that compared with belief neutral inefficiency, U-inefficiency is a stronger concept, since the dominating allocation, $b$, is not allowed to depend on $q$ under U-inefficiency. Equivalently, Uefficiency is a weaker concept than weak belief neutral efficiency.

A consequence of the weak requirements for an allocation to be classified as U-efficient is that the speculative allocation $a_{4}$ in our example is U-efficient. Indeed, as seen in the right panel of Figure 1, for some $q$ 's one agent is better off under $a_{4}$ than under $a_{1}$ and this is also the case when comparing $a_{4}$ with $a_{2}$ and $a_{3}$. In this context, the measure is thus too weak to identify some allocations are reasonable for the SPEPIK to rule out as inefficient.

Going forward, we mainly focus on the IK measure. We introduce a stylized, but still quite general, production economy and study what, if anything, can be said about efficiency and distortions of equilibrium outcomes when using this measure.

\section{The general economy}

\subsection{The Environment}

Consider a two-date economy, $t=0,1$, in which there are $N>1$ agents, with a finite state space $\Omega=\left\{\omega_{1}, \ldots, \omega_{M}\right\}, M>1$. The state is revealed at $t=1$. The information structure is represented by the filtration $\{\mathcal{F}\}_{t}, t=0,1$, where $\mathcal{F}_{0}=\{\emptyset, \Omega\}$ and $\mathcal{F}_{1}=2^{\Omega}$, are the algebras representing information available at $t=0$ and $t=1$, respectively. All random variables throughout the paper are assumed to be $\mathcal{F}_{t}$-adapted.

Agents agree to disagree about the probabilities for different states: Agent $n$ believes that the probability for state $\omega_{m}$ is $q_{m}^{n}$. Agent $n$ 's beliefs can then be represented by a vector $q^{n} \in \mathcal{Q} \stackrel{\text { def }}{=} S^{M}$, with $m$ th element $q_{m}^{n}$. We thus assume that each agent believes that there is a strictly positive probability for each event to occur, which will subsequently allow us to avoid situations where equilibria fail to exist because of the presence of agents who believe there are arbitrage opportunities, and therefore are prepared to take on infinite portfolio positions.

We represent agents' joint beliefs by the vector $\pi=\left(q^{1}, \ldots, q^{N}\right) \in \Pi$, where $\Pi \stackrel{\text { def }}{=}\left(S^{M}\right)^{N}$ is the set of possible joint beliefs. Formally, we associate the probability measure $\mathbb{P}^{n}, n=1, \ldots, N$ with agent $n$, where $\mathbb{P}^{n}\left(\left\{\omega_{m}\right\}\right)=\mathbb{P}\left(\left\{\omega_{m}\right\} ; q^{n}\right)=q_{m}^{n}$, and also the probability space $\left(\Omega, \mathcal{F}_{1}, \mathbb{P}^{n}\right)$. The expectation of a random variable, $\tilde{X}$, given beliefs, $q$, is denoted by $E[\tilde{X} \mid q]$.

The subset of $\Pi$ in which agents have homogeneous beliefs, $\mathbb{P}^{n}\left(\omega_{m}\right)=\mathbb{P}^{n^{\prime}}\left(\left\{\omega_{m}\right\}\right)$ for all $n, n^{\prime}$ and $m$, is $\Pi^{U}$. Here, the $U$-superscript denotes that agents unanimously agree on probabilities. 
We define the bijection $H: \mathcal{Q} \leftrightarrow \Pi^{U}$, by $q \in \mathcal{Q} \leftrightarrow H(q)=(\underbrace{q, q, \ldots, q}_{N}) \in \Pi^{U}$. We also define $\Pi_{q}^{U}=\{H(q)\}$, and we then have $\Pi^{U}=\cup_{q \in \mathcal{Q}} \Pi_{q}^{U}$.

Following Brunnermeier et al. (2013), we define the set of reasonable beliefs as the convex hull of individual agents' beliefs. Specifically, given heterogeneous agent beliefs, $\left(q^{1}, \ldots, q^{N}\right) \in \Pi$, we define the set of reasonable probabilities $\mathcal{Q}_{R}=C H\left(\left\{q^{1}, \ldots, q^{N}\right\}\right)$. As before, the $R$-subscript symbolizes that the set contains the reasonable probabilities or, with a narrower interpretation, that these are the probabilities that a social planner uses when evaluating the efficiency of an allocation. Our analysis will be general, however, allowing a social planner to use a different set of reasonable beliefs. The planner may, e.g., exclude some beliefs that are obviously incorrect.

There is one unit of a divisible and perishable good that can either be consumed at $t=0$ or invested in a linear production technology. A unit invested in the production technology at time 0 yields the random, strictly positive amount, $\tilde{R}$, of the good at $t=1$, at which point it can be consumed. We define $R_{m}=\tilde{R}\left(\omega_{m}\right), m=1, \ldots, M$, the partition of $\Omega, P^{A}=\left\{\tilde{R}^{-1}(\{x\}): x>0\right\}$, and the algebra $\mathcal{F}^{A}=2^{P^{A}}$. We write $R_{B}=R_{m}$, where $\omega_{m} \in B$.

If $\mathbb{P}^{n}=\mathbb{P}^{n^{\prime}}$ on $\mathcal{F}^{A}$ for all pairs of agents $n$ and $n^{\prime}$, i.e., if $\mathbb{P}^{n}(B)=\mathbb{P}^{n^{\prime}}(B)$ for all sets $B \in \mathcal{F}^{A}$, then agents are said to agree on aggregate risk, hence the $A$ superscript. We denote the set of all such beliefs by $\Pi^{A}$. Moreover, if the conditional probabilities, $\mathbb{P}^{n}\left(\omega_{m} \mid B\right)=\mathbb{P}^{n^{\prime}}\left(\omega_{m} \mid B\right)$, for all $B \in P^{A}$, for all agents $n$ and $n^{\prime}$, and states, $\omega_{m}$, then agents are said to agree on idiosyncratic risk, $\pi \in \Pi^{I}$. It follows that $\Pi^{U}=\Pi^{A} \cap \Pi^{I}$, i.e., that agents have homogeneous beliefs if and only if they agree on both systematic and idiosyncratic risk. We note that a special case in which agents agree on aggregate risk is when $\tilde{R} \equiv R$ for some constant $R>0$, i.e., when aggregate returns are risk-free.

Agents' consumptions across time and states are represented by the nonnegative random processes $\tilde{c}^{n}$. Here, agent $n$ 's consumption at time 1 , in state $m$ is $c_{1 m}^{n}$, and at time 0 , it is $c_{0}^{n}$. The joint consumption processes of all agents define the allocation, $a=\left(\tilde{c}^{1}, \ldots, \tilde{c}^{N}\right)$.

Definition 7 (Feasible allocation) An allocation is feasible with aggregate investment, $I \in$ $[0,1]$, if

i) $I=1-\sum_{n=1}^{N} c_{0}^{n}$,

ii) $\sum_{n=1}^{N} c_{1 m}^{n}=I R_{m}, m=1, \ldots, M$.

We do not allow for free disposal. We will assume that agents have strictly increasing utility of consumption, so disposal would never occur even if feasible. The set of feasible allocations is denoted by $\mathcal{A}$. 
Agents have expected utility with Constant Relative Risk Aversion (CRRA) ${ }^{3,4}$

$$
U^{n}=U^{n}\left(a \mid q^{n}\right)=U\left(c_{0}^{n}, \tilde{c}_{1}^{n} \mid q^{n}\right)=\frac{\left(c_{0}^{n}\right)^{1-\gamma}}{1-\gamma}+E_{0}\left[\frac{\left(\tilde{c}_{1}^{n}\right)^{1-\gamma}}{1-\gamma} \mid q^{n}\right], \quad \gamma \neq 1 .
$$

We specifically exclude the case the case of logarithmic utility, $\gamma=1$. As discussed in Section 6 most of our results will not hold when $\gamma=1$ because of well-known special properties of logarithmic utility. We also define the elasticity of intertemporal substitution (EIS), $\psi=\frac{1}{\gamma}$. Note that we for simplicity assume a personal discount rate of zero. All our results hold for positive personal discount rates, so this is without loss of generality.

We say that agents agree on real investment opportunities, $\pi \in \Pi^{\bar{A}}$, if $E\left[\tilde{R}^{1-\gamma} \mid q^{n}\right]=E\left[\tilde{R}^{1-\gamma} \mid q^{n^{\prime}}\right]$ for all $n$ and $n^{\prime}$. Agreeing on real investment opportunities is thus a weaker condition than agreeing on aggregate risk, $\Pi^{A} \subset \Pi^{\bar{A}}$. Such agreement implies that agents agree about how "good" the production technology is (measured in utility terms), but not necessarily on the probabilities for different realizations of productivity.

We define the set of allocations that are efficient for some reasonable belief, $E^{Q}=\cup_{q \in \mathcal{Q}_{R}} E_{q}$. Proposition 1 below shows that the set of IK-efficient allocations in our context has an intuitive characterization, containing precisely those allocations that are efficient for some reasonable belief. The proposition also guarantees that the IK-efficient set is not empty, which is not immediate since IK-dominance is in general not an acyclical relation.

Proposition 1 IK-efficiency is equivalent to efficiency for some $q \in \mathcal{Q}_{R}, E^{I K}=E^{Q}$,

\subsection{Competitive equilibrium}

We study allocations that may arise as outcomes of competitive equilibrium. To this end, we assume that each agent has access to the production technology, and is endowed with a strictly positive initial amount of the good, $K^{n}>0$, and normalize the total supply of the good to unity, $\sum_{n=1}^{N} K^{n}=1$. We represent this initial allocation as a vector, $K=\left(K^{1}, \ldots, K^{N}\right) \in \mathcal{K}$, where $\mathcal{K}=S^{N}$. The primitives of the economy can now be summarized by the tuple $\mathcal{E}=(\gamma, \Omega, \pi, K, \tilde{R})$.

At $t=0$, agents can trade in a market for state-contingent Arrow Debreu (AD) securities on each state. The price of an AD security that pays a unit of the good at time 1 in state $m$, measured in time- 0 units of the good, is $p_{m}>0$. The absence of arbitrage implies that

$$
\sum_{m} p_{m} R_{m}=1
$$

\footnotetext{
${ }^{3}$ We will subsequently discuss extensions to more general utility specifications.

${ }^{4}$ Here, the $\tilde{c}_{1}^{n}$ dependence of $U\left(c_{0}^{n}, \tilde{c}_{1}^{n} \mid q^{n}\right)$ represents that $U$, being a time-0 expectation, and hence $\mathcal{F}_{0}$-measurable, is a function of the future realization of $\tilde{c}_{1}^{n}$ in all states, $c_{11}^{n}, c_{12}^{n}, \ldots, c_{1 M}^{n}$. It does not mean that $U$ is a function of the realization of $\tilde{c}_{1}^{n}$ (which is of course not $\mathcal{F}_{0}$-measurable).
} 
since agents would otherwise form arbitrage portfolios in $\mathrm{AD}$ securities and the real asset that represents the production technology. ${ }^{5}$

The optimization problem for agent $n$ is then

$$
\begin{array}{rl}
\max _{d_{1}^{n}, \ldots, d_{M}^{n}} & U\left(c_{0}^{n}, \tilde{c}_{1}^{n} \mid q^{n}\right), \\
c_{0}^{n}= & K^{n}-\sum_{i=1}^{M} d_{m}^{n} p_{m} \\
c_{1 m}^{n}= & d_{m}^{n} .
\end{array}
$$

Here, $d_{m}^{n} \in \mathbb{R}$ is agent $n$ 's demand for the $m$ th $\mathrm{AD}$ security, and we define the demand vector $d^{n}=\left(d_{1}^{n}, \ldots d_{M}^{n}\right) \in \mathbb{R}^{M}$. We restrict our attention to the case with strictly positive AD security prices, since arbitrage opportunities would arise if some prices were nonpositive, inconsistent with equilibrium. We define the state-price vector $p=\left(p_{1}, \ldots, p_{M}\right) \in \mathcal{P}$, where $\mathcal{P}=\mathbb{R}_{++}^{M}$.

The strict concavity of agents' preferences implies that $d^{n}$ is a unique and smooth function of $K^{n}, q^{n}$, and $p$, so we can write

$$
d^{n}=D\left(K^{n}, q^{n} \mid p\right),
$$

for a smooth function, $D$. We now have:

Definition 8 A state-price vector, $p$, such that

$$
\begin{gathered}
\sum_{m} p_{m} R_{m}=1, \quad \text { and } \\
\sum_{n}\left(D\left(K^{n}, \pi^{n} \mid p\right)\right)_{m}=I R_{m}, \quad m=1, \ldots, M,
\end{gathered}
$$

for some $I \in[0,1]$, defines a (Walrasian) equilibrium, given initial allocations, $K \in \mathcal{K}$, and beliefs, $\pi \in \Pi$.

We have the following existence and uniqueness result:

Proposition 2 In the economy, $\mathcal{E}=(\gamma, \Omega, \pi, K, \tilde{R})$, there exists an equilibrium state-price vector, p. Moreover, there exists a $\underline{\gamma}<1$, such that $p$ is unique if $\gamma>\underline{\gamma}$.

Here, $\underline{\gamma}$ is allowed to depend $K, \pi$ and $\tilde{R}$. In our subsequent analysis, we assume that $\gamma \geq \bar{\gamma}$ so that equilibrium is unique, thus excluding economies with very low $\gamma$ 's. This allows us to use functions instead of the more cumbersome use of correspondences. The state price vector is then a function of $K$ and $\pi, p(K, \pi)$, (taking $\gamma$ and $R_{1}, \ldots, R_{M}$ as given), as are investments and consumption:

$$
I(K, \pi), \quad c_{0}^{n}(K, \pi), \quad \tilde{c}_{1}^{n}(K, \pi), \quad n=1, \ldots, N .
$$

\footnotetext{
${ }^{5}$ Strictly speaking, this argument only works for interior allocations, such that $0<I<1$, a condition that will always hold in equilibrium in our economy since our utility specification satisfies the Inada conditions.
} 
Aggregate consumption is $\tilde{C}(K, \pi)=\sum_{n} \tilde{c}^{n}$, and we note that the budget constraint implies that

$\tilde{C}_{1}=I \tilde{R}$. We write the mapping from agents' beliefs and initial capital to the equilibrium outcome in terms of allocation and prices $(a, p) \in \mathcal{A} \times \mathcal{P}$, as $(a, p)=G(K, \pi)$.

\subsection{Introducing idiosyncratic endowment shocks}

We introduce the additional assumption that agents are exposed to idiosyncratic endowment shocks. Each agent faces a shock, $\tilde{e}^{n}$, at $t=1$, where $e_{m}^{n}$ is the size of agent $n$ 's shock in state $m$. These shocks are idiosyncratic, i.e., they are such that

$$
\sum_{n=1}^{N} e_{m}^{n}=0, \quad m=1, \ldots, M
$$

The endowment shocks are summarized in the vector $e=\left(e_{0}^{1}, e_{0}^{2}, \ldots, e_{0}^{N}, e_{11}^{1}, \ldots, e_{11}^{N}, e_{12}^{1}, \ldots, e_{1 M}^{N}\right) \in$ $\mathbb{R}^{N \times(M+1)}$.

In the complete market equilibrium, these shocks affect agents' wealths. We need to ensure that each agents' total wealth, including the value of endowment shocks, is strictly positive for equilibrium to be well-defined. We ensure this by the following extension: Given the equilibrium state price vector in an economy with no endowment shocks and initial allocation $K$, this state price vector also determines an equilibrium in any economy with initial endowments $K^{\prime}$, and endowment shocks $e$, such that

$$
K^{n}=\left(K^{\prime}\right)^{n}+\sum_{m} e_{m}^{n} p_{m}, \quad n=1, \ldots, N
$$

in which each agent, $n$, solves the optimization problem

$$
\begin{array}{rl}
\max _{d_{1}^{n}, \ldots, d_{M}^{n}} & U\left(c_{0}^{n}, \tilde{c}_{1}^{n} \mid q^{n}\right), \\
c_{0}^{n}= & \left(K^{\prime}\right)^{n}-\sum_{i=1}^{M} d_{m}^{n} p_{m} \\
c_{1 m}^{n} & =d_{m}^{n}+e_{m}^{n}
\end{array}
$$

Our analysis covers this extended set of economies with idiosyncratic shocks, summarized by the tuple $\mathcal{E}=(\gamma, \Omega, \pi, K, \tilde{R}, e)$. A sufficient condition for agent wealth to be strictly positive in the economy with endowment shocks is that $K^{n} R_{m}+e_{m}^{n}>0$ for all $n$ and $m$.

The following result shows that, in contrast to the case of homogeneous beliefs, equilibrium is always IK-inefficient under heterogeneous beliefs:

Proposition 3 Within the production economy, $\mathcal{E}$, equilibrium is IK-efficient if and only if beliefs are homogeneous, $\pi \in \Pi^{U}$.

Thus, just as in the exchange economy setting, as discussed in Brunnermeier et al. (2013), Gilboa 
et al. (2013), Gayer et al. (2013), and Blume et al. 2014, the friction free equilibrium outcome is inefficient under heterogeneous beliefs in the production economy.

\section{Equilibrium distortions}

To understand the sources of IK-inefficiency, we make a decomposition into a speculative component and an investment distortion component, respectively. Speculative distortions correspond to pure transfers between agents across states, and also arise in the exchange economy, whereas investment distortions have real effects and only arise in the production economy. Unsurprisingly, speculative distortions always arise when agents have heterogeneous beliefs. In many cases the presence and directions of investment distortions can also be characterized, which is a priori more surprising.

We define a unanimous distortion of an outcome in the same spirit as when defining U-efficiency. For an outcome to be classified as unanimously distorted, it must be distorted under any reasonable belief. Specifically, consider the set of equilibrium outcomes supported by reasonable beliefs,

$$
\Gamma^{U}=\left\{G(K, \pi): K \in \mathcal{K}, \pi \in \cup_{q \in \mathcal{Q}_{R}} \Pi_{q}^{U}\right\}
$$

a characteristic, $v$, that is defined by the function, $v=f(a, p)$, where $f: \mathcal{A} \times \mathcal{P} \rightarrow \mathbb{R}$, and furthermore define the set of (unanimously) reasonable values of $v, F_{v}^{U}=\left\{f(a, p):(a, p) \in \Gamma^{U}\right\}$.

Definition 9 The variable, $v$ is said to be Unanimously Distorted in equilibrium if $v \notin F_{v}^{U}$. It is too low if $v<\inf F_{v}^{U}$, and too high if $v>\sup F_{v}^{U}$.

Note that a distortion is defined in the context of an equilibrium outcome, $(a, p)$. Alternatively, we could have defined a distortion with respect to the real allocation, $a$, alone. Since we know from the second welfare theorem that any efficient allocation can be generated (in a homogeneous beliefs economy) as an equilibrium outcome by appropriately choosing agents' initial allocations, our definition encapsulates such an allocation-oriented definition. The advantage of our definition is that it allows for the study of both financial (price) and real (allocation) distortions within a unified framework.

\subsection{Speculation and investment distortions}

We first use the distortion concept to introduce a precise statement of what it means for speculation to be present in a complete market equilibrium. As discussed in Hirshleifer (1975), the term speculation has been associated with the presence of risk transfer between risk tolerant and risk averse agents, following arguments made by Keynes (1930) and Hicks (1946), as well as with the concept of trades that are generated from agents' different beliefs and information, in line with the view of Working (1953). Here, we focus on Working's interpretation. Within our setting, the variation of an agent's share of total consumption across states in the complete market equilibrium is 
always generated by differences in beliefs, and we use this variation as the basis to define speculation:

Definition 10 The speculative component of the complete market equilibrium outcome is

$$
s=\frac{1}{N(M+1)} \sum_{n=1}^{N}\left(\left|\frac{c_{0}^{n}}{C_{0}}-\eta^{n}\right|+\sum_{m=1}^{M}\left|\frac{c_{1 m}^{n}}{C_{1 m}}-\eta^{n}\right|\right),
$$

where $\eta^{n}=\frac{1}{M+1}\left(\frac{c_{0}^{n}}{C_{0}}+\sum_{m=1}^{M} \frac{c_{1}^{n}}{C_{1 M}}\right)$ is agent $n$ 's mean consumption share across states and time.

In any complete market homogeneous beliefs economy, the speculative component is zero, so its only unanimously reasonable value is zero, $F_{s}^{U}=\{0\}$. An economy with $s>0$ is therefore (unanimously) speculatively distorted, in line with Definition 9. The following result shows that speculative distortions always arise under heterogeneous beliefs in equilibrium:

Proposition 4 If agents disagree, $\pi \notin \Pi^{U}$, equilibrium is speculatively distorted.

We next study the investment distortions that may arise in equilibrium. In line with Definition 9, aggregate investments are unanimously distorted if they cannot be supported in any homogeneous beliefs economy for some reasonable belief, $q \in \mathcal{Q}_{R}$. If equilibrium investments are lower than investments in any such homogeneous beliefs economy, there is underinvestment, and if they are higher there is overinvestment.

The following proposition shows that the set of reasonable investment levels is small when agents agree on aggregate risk:

Proposition 5 If agents agree on aggregate investment opportunities, $\pi \in \Pi^{\bar{A}}$, the set of reasonable investments contains a single element, $F_{I}^{U}=\left\{I^{*}\right\}$.

As a consequence of agents' agreement about the reasonable investment level, we may expect aggregate investments to be reasonable in equilibrium. However, this is not the case, as shown by the following result:

Proposition 6 If agents agree on aggregate investment opportunities but disagree on idiosyncratic risk, $\pi \in \Pi^{\bar{A}} \backslash \Pi^{I}$, equilibrium investments are unanimously distorted.

Thus, the effects on investments of heterogeneous beliefs are straightforward: disagreement about idiosyncratic risk unanimously leads to distortions.

The source of the unanimous investment distortion is a wealth effect: When agents disagree, they all feel wealthier because of their expected gains from speculation. The effect on investments of increased wealth is known to be intimately connected to the elasticity of intertemporal substitution, $\psi$, and it is therefore not surprising that the direction of the investment distortion is too: 


\section{Proposition 7}

If agents agree on aggregate investment opportunities but disagree on idiosyncratic risk, $\pi \in$ $\Pi^{\bar{A}} \backslash \Pi^{I}$, then

- if the elasticity of intertemporal substitution, is greater than one, $\psi>1$, there is overinvestment in equilibrium, $I>I^{*}$.

- if the elasticity of intertemporal substitution is less than one, $\psi<1$, there is underinvestment in equilibrium, $I<I^{*}$.

In equilibrium, all agents have higher expected utility (given their beliefs) than in the homogeneous beliefs economy, because they expect gains from speculation on idiosyncratic risk. They therefore readjust their consumption and investments at $t=0$ to take this higher utility into account, decreasing investments if $\psi<1$ and increasing investments if $\psi>1$. The distortion is thus a consequence of "spurious unanimity" (see Mongin 1995) among agents: Agents believe the distorted investment outcome to be optimal for themselves, although they base their conclusions on different - mutually exclusive - beliefs. It follows from Proposition 1 that IK-efficient outcomes are neither speculatively, nor investment distorted:

Corollary 1 An allocation is IK-efficient, $a \in E^{I K}$, if and only if it is neither speculatively distorted, nor investment distorted.

Example 1 Consider an economy with three agents, $\gamma=2$, and corresponding EIS, $\psi=\frac{1}{2}$. There are four states in which investment returns are $R_{1}=1, R_{2}=1, R_{3}=1.4$, and $R_{4}=1.4$, respectively, and no endowment shocks. Note that returns are the same in state 3 and 4, representing purely idiosyncratic risk. Table 2 shows the agents' beliefs about the probabilities for the states. Figure 2 shows equilibrium investments (horizontal axis) and speculative component (vertical axis)

\begin{tabular}{l|r|r|r}
\hline Agent & 1 & 2 & 3 \\
\hline State 1 & 0.1 & 0.25 & 0.4 \\
State 2 & 0.1 & 0.25 & 0.4 \\
State 3 & 0.4 & 0.25 & 0.01 \\
State 4 & 0.4 & 0.25 & 0.19 \\
\hline
\end{tabular}

Table 2: The table shows the beliefs of agent $n=1,2,3$ for the probability of each of states $m=1,2,3,4$ to occur.

for all equilibrium outcomes, for different initial allocations of capital, $K \in \mathcal{K}$.

Several observations are in order: The speculative component approaches zero at three points: $I=0.4694, I=0.4847$ and $I=0.4984$, corresponding to initial allocations where all capital is 
given to agent 1, 2, and 3, respectively. Since we assume strictly positive initial capital for each agent, these limit points are not part of the set of equilibrium outcomes.

The set of reasonable investments is $F_{I}^{U}=[0.4694,0.4984]$, is represented by the dotted (red) line on the horizontal axis. This line also represents the set of IK-efficient allocations. Any value of I outside of this interval is distorted. There is a nonempty set of equilibria with underinvestment $(I<0.4694)$, but no equilibrium with overinvestment. This is in line with Proposition 7 , since the $E I S, \psi$, is less than one. Moreover, there is also a nonempty set of equilibria for which investments are not distorted, which is also in line with Proposition 7 , since agents also disagree on aggregate investment opportunities in this example.

We note that there are no belief neutrally efficient allocations, again suggesting that the concept is too strong in this setting. Specifically, any investment level is inefficient for some $q \in \mathcal{Q}_{R}$, causing the efficiency set to be empty. Moreover, U-efficiency is too weak to rule out any equilibrium allocations - the whole equilibrium region is U-efficient-as shown by the following result:

Proposition 8 Any equilibrium outcome in the heterogeneous beliefs economy is U-efficient.

Proposition 8 immediately implies that no equilibrium outcome, is ever Unanimity Pareto dominated by any feasible allocation.

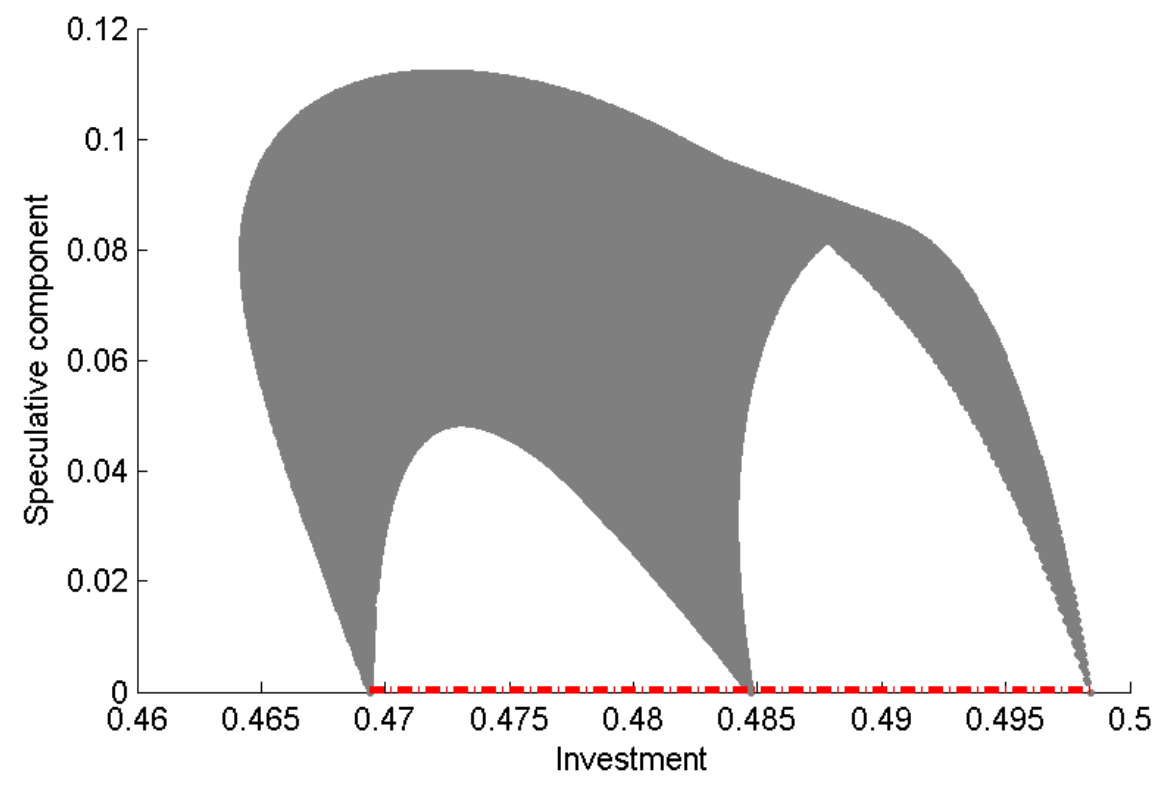

Figure 2: Equilibrium distortions. The figure shows the possible investment (horizontal) and speculative (vertical) equilibrium outcomes, for all different initial allocations, $K \in \mathcal{K}$ in the example described in Table 2. 


\subsection{Price Distortions}

We next study the financial side of the economy. Along similar lines as in our earlier analysis, we are especially interested in understanding under what conditions there is unanimous agreement about there being equilibrium price distortions, i.e., mispricing. For a general vector $a \in \mathbb{R}^{M}$, we let $a$ represent the asset that pays $a_{m}$ in state $m$, and $P^{a}=\sum_{m} a_{m} p_{m}$ its corresponding equilibrium price, and similarly for the vector $b \in \mathbb{R}_{M}$ with price $P^{b}$. The AD security that pays a unit of the good in state $m$ is $\delta_{m}$ and, more generally, a security that pays a unit in the set of states, $B \subset \Omega$, is $\delta_{B}$.

\section{Definition 11}

1. There is mispricing in equilibrium, if there exist assets, $a \in \mathbb{R}^{M}$ and $b \in \mathbb{R}^{M}$, such that $P^{b} \neq 0$, and $\frac{P^{a}}{P^{b}}$ is distorted. In this case, we say that there is $(a, b)$ mispricing.

2. There is intratemporal mispricing if there is $\left(\delta_{i}, \delta_{j}\right)$ mispricing, for some AD securities, $\delta_{i}$, $\delta_{j}, 1 \leq i, j \leq M$.

3. There is intertemporal mispricing if there is $(a, \tilde{R})$ mispricing for some asset a.

4. The risk-free rate is distorted if there is $(\mathbf{1}, \tilde{R})$ mispricing.

The asset $b$ has the natural interpretation of being the numeraire, i.e., mispricing is defined in terms of the price of asset $a$ measured in number of units of asset $b$. When $b=\tilde{R}$, this numeraire is in terms of $t=0$ consumption, since $P^{\tilde{R}}=1$. We use the term intratemporal mispricing for the case when we compare the relative prices of two AD securities - which both make their payments at $t=1$. Intertemporal mispricing, on the the other hand, identifies mispricing in terms of units of current consumption, since the numeraire $b=\tilde{R}$ with price $P^{b}=1$ is chosen.

The existence of mispricing in a market could potentially be used by a social planner to detect real and speculative distortions, so it is important to understand under which conditions, and for which types of assets, it occurs. Of course, if agents agree on all risk, there will never be mispricing, so the presence of speculative distortions is a necessary condition for mispricing. However, the reverse is not true. It is straightforward to come up with examples where there are speculative distortions - due to disagreement about idiosyncratic or aggregate risk, or about both-but no mispricing. Similarly there are examples with equilibrium investment distortions and mispricing, as well as examples with investment distortions and no mispricing. In general, the links between financial and real distortions is therefore not very strong.

Our first result rules out intratemporal mispricing completely:

Proposition 9 There is never intratemporal mispricing in equilibrium.

The economic intuition for this result is that the relative prices between two AD securities will be determined by an average of the agents' different beliefs about the relative likelihood of these two 
events occurring, together with the aggregate marginal rate of substitution between the two states (about which there is no disagreement) and will therefore be consistent with some reasonable belief. The result shows that the relative prices of such contingent claims that make their payments at the same point in time - even if there is a lot of disagreement about their likelihoods - cannot be used to detect distortions in the economy under an EPIK measure.

In contrast, intertemporal mispricing occurs generically, as long as the economic environment is rich enough to have many states and agents with sufficiently different beliefs. Indeed, although it is possible to design examples in which there is no equilibrium mispricing even in such environments, these examples are the rare exceptions. The argument is made precise in the following proposition:

Proposition 10 Consider an economy in which there are more states than agents, $M>N$, and define the matrix $A \in \mathbb{R}_{++}^{M \times N}$, with elements $A_{m n}=\left(q_{m}^{n}\right)^{1 / \gamma}$. Assume that the rank of $A$ is $N$. Then, for all Pareto weights, $\lambda \in S^{N}$, except possibly for a subset of Lebesgue measure zero, there is intertemporal mispricing in equilibrium.

The proof of Proposition 10 proceeds by constructing an asset that all agents agree upon the price of, which is possible given that the set of assets is so rich $(M>N)$, and then showing that the equilibrium price of this asset is typically nonzero, except possibly in some knife-edge cases. That asset's price is thus typically distorted in equilibrium.

The result is general, but the argument is somewhat technical and thereby provides quite limited insight about where in the asset space one should look for mispricing. The following proposition provides more insight, being based on economic arguments:

Proposition 11 If for some idiosyncratic event, $B \in P^{A}$, containing at least three states, all agents agree about $\mathbb{P}\left(\omega_{m} \mid B\right)$, for some $\omega_{m}$, but there is disagreement about some other state in $B$ between at least two agents, then there is $\left(\delta_{m}, \delta_{B}\right)$ mispricing in equilibrium.

The intuition behind the result is that unanimously agreed upon mispricing is difficult to identify in states that agents disagree upon, since such states have a wide range of reasonable prices. On the other hand, for a state $\omega_{m}$, for which there is no disagreement, all agents also agrees on the ratio, $k$, of the values of $\delta_{m}$ and $\delta_{B}$. This is the first part of the argument. The second part, that in equilibrium $\frac{p^{m}}{p^{B}} \neq k$, follows from the fact that since agents do not see any speculative benefits in state $m$, but do see such benefits in some of the other states in $B$, this moves their demands away from asset $\delta_{m}$, thereby leading to mispricing. The lesson to be drawn is that it may be easiest to identify mispricing by studying the prices of assets about which there is the least disagreement.

\section{$5 \quad$ Policy implications}

It follows from Corollary 1 that a planner who wishes to obtain an IK-efficient outcome should avoid allocations that lead to either speculative or investment distortions. In an exchange economy 
with no endowment shocks, it may be relatively simple to avoid speculative distortions by simply shutting down speculative markets. However, when endowment shocks are present, even shutting down markets for purely idiosyncratic risk may be costly because of lost hedging opportunities. A similar argument holds for the effects of introducing a tax on trades in such markets (a Tobin tax), as shown in the following example.

Example 2 Consider the two agent, two state, economy in which initial allocations are $K=$ $(1 / 2,1 / 2), \gamma=2$, and $R_{1}=R_{2}=1$. Agents are exposed to idiosyncratic endowment shocks, $e_{1}^{1}=e_{2}^{2}=1 / 2, e_{2}^{1}=e_{1}^{2}=-1 / 2$, and heterogeneous beliefs, $q_{1}^{1}=q_{2}^{2}=7 / 10, q_{2}^{1}=q_{1}^{2}=3 / 10$. It is easy to verify that the IK-efficient allocations are those for which each agent's consumption is the same across time and state: $c_{0}^{1}=c_{11}^{1}=c_{12}^{1}$, and $c_{0}^{2}=c_{11}^{2}=c_{12}^{2}$ and consequently the efficient investment level is $I^{*}=1 / 2$.

In equilibrium with no taxes, consumption is $c_{0}^{1}=c_{0}^{2}=0.253, c_{11}^{1}=c_{22}^{2}=0.299$, and $c_{12}^{1}=c_{21}^{2}=$ 0.196, an IK-inefficient allocation which contains both speculation and underinvestment. Because of symmetry, it is also clear that the state prices are $p_{1}=p_{2}=1 / 2$.

Now consider a transaction tax that is proportional to the face value of traded AD securities, i.e., the total price agent $n$ pays for the demand $d_{m}^{n}$ of $A D$ security $m$, is $d_{m}^{n} p_{m}+\tau\left|d_{m}^{n}\right|$. The tax is revenue neutral, which is achieved by the government making a lump-sum payment of $X$ to each agent at $t=0$. Agent $n$ thus solves the optimization problem:

$$
\max _{I^{n}, d_{1}^{n}, d_{2}^{n}} \frac{\left(K^{n}+X-I^{n}-\tau \sum_{m}\left|d_{m}^{n}\right|\right)^{1-\gamma}}{1-\gamma}+\sum_{m} q_{m}^{n} \frac{\left(e_{m}^{n}+I^{n}+d_{m}^{n}\right)^{1-\gamma}}{1-\gamma} \text {, s.t. } \sum_{m} d_{m}^{n} p_{m}=0 .
$$

An equilibrium is then defined as a state price vector that yields market clearance, $\sum_{n} d_{m}^{n}=0$, $m=1, \ldots, M$, and government payments that are equal to tax revenues, $N X=\tau \sum_{n, m}\left|d_{m}^{n}\right|$.

In this example, it is easy to verify that equilibrium with the tax is such that again $p_{1}=$ $p_{2}=\frac{1}{2}$, and that speculative distortions are strictly increasing in $\tau$, making any equilibrium with a transaction tax strongly IK-dominated by the friction-free equilibrium. The reason the transaction tax in this example decreases welfare is because it makes it more costly for agents to hedge their endowment shocks, and therefore leads to less risk sharing than without the tax.

A key takeaway of the previous example is thus that when both hedging and speculation motives are present, it may be difficult to address speculative distortions by imposing a transaction tax or shutting down markets. ${ }^{6}$ The equilibrium investment distortion may actually be easier to address by a social planner, given that the economy's EIS, $\psi$, is known. A short summary of empirical evidence about the EIS reveals two stylized facts: First, various studies in different markets suggest that the EIS is lower than one; for a recent survey, see Havranek et al. (2013). Second, the EIS is higher in markets with more sophisticated and wealthier investors. For example, Vissing-Jorgensen (2002),

\footnotetext{
${ }^{6}$ Dávila (2014) shows that as long as agents who are optimistic about a financial asset are on average long the asset, whereas those who are pessimistic are on average short, there is a strictly positive transaction tax that is welfare improving. Our example does not satisfy this condition, since the endowment shocks are such that the agents wish to hedge the $\mathrm{AD}$ securities they are optimistic about.
} 
shows that the EIS implied from agents' Euler equations is consistently higher for bond market participants than for stock market participants and, in turn, higher for stock market participants than for nonparticipants. Blundell et al. (1994) find that the EIS is higher for rich than for poor households, and in a meta study Havranek et al. (2013) find that the EIS is higher in wealthier countries than in poor countries. We therefore focus on the case when the EIS is less than one. The analysis when $\psi>1$ is identical, the only difference being that the signs of taxes and subsidies switch.

Example 3 Assume that the social planner introduces an investment tax, such that agents need to invest $(1+\tau) I$ at $t=0$ to receive $I \tilde{R}$ at $t=1$. Again, the tax is revenue neutral. The government makes a lump-sum payment, $X$, such that in equilibrium the tax matches the payment. Agent $n$ 's optimization problem is then

$$
\max _{I^{n}, d_{1}^{n}, d_{2}^{n}} \frac{\left(K^{n}+X-(1+\tau) I^{n}\right)^{1-\gamma}}{1-\gamma}+\sum_{m} q_{m}^{n} \frac{\left(e_{m}^{n}+I^{n}+d_{m}^{n}\right)^{1-\gamma}}{1-\gamma}, \text { s.t. } \sum_{m} d_{m}^{n} p_{m}=0
$$

and in equilibrium $\sum_{n} d_{m}^{n}=0, m=1, \ldots, M$, and $N X=\tau I$. In the economy with the same parameters as in Example 2, one can verify that by choosing $\tau=0.0145$ the social planner obtains undistorted equilibrium investments, $I=I^{*}=0.5$. It is also straightforward to verify that the allocation with the investment tax dominates the no-tax outcome under Pareto weights $\lambda=(1 / 2,1 / 2)$,

for all reasonable beliefs. In other words, the equilibrium outcome with the investment tax weakly IK-dominates the no-tax outcome.

In this example, the speculative distortion is still present, investment distortions are avoided by imposing the tax. The following proposition shows that such improvements are in general possible, when disagreement is about idiosyncratic risk.

Proposition 12 In an economy with disagreement about idiosyncratic risk and agreement about aggregate risk, $\pi \in \Pi^{A} \backslash \Pi^{I}$, and $\psi<1$, there is a strictly positive investment tax, such that the equilibrium outcome in the economy with the investment tax weakly IK-dominates the equilibrium outcome in the economy without the tax.

Thus, addressing investment distortions is indeed in some cases easier for the social planner than addressing speculative distortions.

\section{Extensions}

Our model is perfectly general with respect to the state space structure, endowment shocks, and beliefs, but makes fairly strong assumptions about agent preferences - assuming that all agents have identical CRRA preferences - and about the production technology - assuming that only one technology is available. We discuss how these assumptions may be relaxed. 


\subsection{Alternative preferences}

For tractability, we have assumed CRRA preferences, but our results about the speculative inefficiency of equilibrium under heterogeneous beliefs hold much more generally. As long as corner solutions can be ruled out, e.g., by imposing Inada conditions on utility function, Propositions 3 and 4 extend to general, agent specific von-Neumann Morgenstern expected utility specifications: $U^{n}=u^{n}\left(c_{0}\right)+E_{0}^{n}\left[v^{n}\left(\tilde{c}_{1}^{n}\right) \mid q^{n}\right]$. The only modification needed is that speculation needs to be defined over marginal utilities, not over consumption shares. Only for CRRA preferences are the two definitions equivalent.

The investment distortions results also generalize outside of the CRRA framework, but the EIS needs to be well defined. It is, e.g., straightforward to show that Propositions 5-7 also hold when agents have Epstein-Zin utility,

$$
\begin{aligned}
U^{n}=U\left(c_{0}^{n}, \tilde{c}_{1}^{n}, \pi_{n}\right) & =\left(\left(c_{0}^{n}\right)^{\frac{1-\gamma}{\theta}}+E\left[\left(\tilde{c}_{1}^{n}\right)^{1-\gamma} \mid \pi_{n}\right]^{\frac{1}{\theta}}\right)^{\frac{\theta}{1-\gamma}} \\
& =\left(\left(c_{0}^{n}\right)^{\frac{1-\gamma}{\theta}}+\left(\sum_{m=1}^{M}\left(c_{1 m}^{n}\right)^{1-\gamma} \pi_{m}^{n}\right)^{\frac{1}{\theta}}\right)^{\frac{\theta}{1-\gamma}}
\end{aligned}
$$

Here, the variable $\gamma$ represents risk-aversion as in section 3.1. However, $\gamma$ does no longer play the dual role of risk aversion and reciprocal of the EIS. With Epstein-Zin utility we have the additional parameter $\theta=\frac{1-\gamma}{1-\frac{1}{\psi}}$, where $\psi$ is the elasticity of intertemporal substitution (EIS). When $\psi=\frac{1}{\gamma}$, $\theta=1$, and this utility specification reduces to expected utility with power preferences. When $\theta \neq 1$, $\psi$ will govern whether disagreement leads to under- or over-investment in equilibrium.

We have specifically excluded the case of logarithmic utility $(\gamma=1)$ throughout the paper. Under logarithmic utility, speculative distortions arise in equilibrium, but never investment distortions, nor mispricing, because of the well-known property that income and substitution effects cancel out under a log utility.

\subsection{Pure exchange economy}

It is interesting to compare our result to those that arise in the exchange economy, i.e., when the number of production technologies available is zero. The economic setup in this case is similar to that in Section 3.1 with the exception that agents cannot invest. Instead, they receive endowments $e^{n}=\left(e_{0}^{n}, e_{11}^{n}, \ldots, e_{1 m}^{n}\right)$, and the total endowment is $e=\sum_{n=1}^{N} e^{n}$. Given a state price vector, $p$, the optimization problem of agent $n$ is then

$$
\begin{array}{rl}
\max _{c_{0}^{n}, \tilde{c}_{1}^{n}} & U\left(c_{0}^{n}, \tilde{c}_{1}^{n} \mid q^{n}\right) \\
c_{0}^{n}+\sum_{m=0}^{M} p_{m} c_{1 m}^{n} & =e_{0}^{n}+\sum_{m=0}^{M} p_{m} e_{1 m}^{n} .
\end{array}
$$


Similar arguments as those leading to Proposition 4 implies the existence of a unique equilibrium state-price vector.

Obviously, in this setting there can be no investment distortions. Moreover, an identical argument as in Proposition 4 implies that speculative distortions always arise when agents disagree. With respect to mispricing, there are difference compared with the production economy, as shown by the following result:

Proposition 13 In the production economy without aggregate risk, the risk-free rate is never distorted. In the exchange economy without aggregate risk, the risk-free rate is always distorted.

The reason for the difference is that in a production economy, agents change their investments to smooth out perceived gains from speculation across time. In a pure exchange economy, however, this is not possible. Instead, the prices have to change to keep aggregate consumption equal to total endowments. A similar mechanism is explored in Ehling et al. (2013).

\subsection{Multiple production technologies}

Consider the case with $2 \leq J \leq M$ linear production technologies. Each unit invested in technology $0 \leq j \leq J$ at time 0 yields a strictly positive amount $R_{1 m}^{j}$ at time 1 in state $m=1, \ldots, M$. With a slight abuse of notation, let $R$ be the $J \times M$ matrix with element $R_{j, m}=R_{1 m}^{j}$, i.e., $R_{j, m}$ is the payout of technology $j$ in state $m$. We assume that the rank of $R$ is $J$ so no technology can be replicated as linear combinations of the other technologies. The next proposition characterizes a set of asset prices that are never distorted.

Proposition 14 Let $\tilde{X}$ denote a random payoff at time 1 and $P(\tilde{X})$ denote the equilibrium price of an asset that generates this payoff. If $\tilde{X} \in \operatorname{span}(R)$ then $P(\tilde{X})$ is not mispriced.

Proposition 14 shows that asset that are in the span of the payoff matrix of the $J$ technologies cannot be mispriced in equilibrium. If this was the case there would be arbitrage opportunities. This extends the result that the price of the production technology in section 3.1 can never be mispriced. The general implication is that the scope for mispricing decreases when the number of production technologies increases, exemplified by the comparison between the exchange economy and the production economy, where disagreement about idiosyncratic risk led to a distorted riskfree rate in the former but not in the latter. The following result is an immediate consequence of Proposition 14, implying that when there are as many linearly independent technologies as states, Arrow-Debreu prices are completely determined by the marginal rate of technical substitution:

Corollary 2 If $J=M$ there is no mispricing in equilibrium. 


\section{Concluding remarks}

The analysis of efficiency and distortions in economies with heterogeneous beliefs introduces multiple challenges, especially when agents wish to hedge risks, productive resources need to be allocated, and the social planner has incomplete knowledge about true probabilities. Even defining what constitutes an inefficiency or distortion, in a manner that is operational for the social planner, poses a challenge in itself. Characterizing conditions under which inefficiencies, mispricing or distortions unambiguously occur in equilibrium poses another challenge, given the range of reasonable probabilities that must be considered. Defining actions that may be taken by the social planner, that balance the benefits of reduced speculation against the potential costs of decreased hedgeing opportunities and distorted productive allocations poses a third.

Our model, although necessarily very stylized, provides a setting within which each of these challenges can be constructively addressed. The concepts of IK-inefficiency and unanimous distortions allow for surprisingly strong equilibrium characterizations, both of real (investment) and of financial (pricing) variables, although based on very weak information assumptions. As a practical application, such results may, for example, have allowed for an objective discussion about whether the degree of resources funneled to New Economy startups during the high-tech boom of the late 1990's was appropriate, without taking a stand on the prospects of the New Economy sector as a whole. Potential policy responses to inefficiencies and distortions are also relatively straightfor-

ward to analyze within the model. Altogether, we view this as a fruitful step toward a more general theory of efficiency and distortions under heterogeneous beliefs. 


\section{A Proofs}

Proof of Proposition 1

We note that each $a \in E^{Q}$ is associated with a unique pair of investment level and Pareto weight, $(I, \lambda)$, i.e., $a^{\prime} \neq a \Leftrightarrow\left(I^{\prime}, \lambda^{\prime}\right) \neq(I, \lambda)$, and that $\forall b \in \mathcal{A}$, such that $b \neq a, a \succ_{q}^{\lambda} b$.

For each $q \in \mathcal{Q}_{R}$, we denote the ( $\lambda$-independent) optimal investment level by $I_{q}$, and define $F_{I}^{U}=\cup_{q \in \mathcal{Q}_{R}}\left\{I_{q}\right\}$. We note that $F_{I}^{U}$ is a closed interval.

The optimal allocation given $q$ is characterized by the allocation, $a$, such that

$$
\begin{aligned}
\frac{1}{v^{n}} c_{0}^{n} & =\frac{1}{v^{n^{\prime}}} c_{0}^{n^{\prime}}, \\
\frac{1}{v^{n}} c_{1 m}^{n} & =\frac{1}{v^{n^{\prime}}} c_{1 m}^{n^{\prime}}, \quad m=1, \ldots, M,
\end{aligned}
$$

$I_{q}=1-C_{0}=1-\sum_{n} c_{0}^{n} \in F_{I}^{U}$, and vector of consumption weights, $v^{n}=\frac{\left(\lambda^{n}\right)^{1 / \gamma}}{\sum_{n}\left(\lambda^{n}\right)^{1 / \gamma}} \in S^{N}$.

We first show that $E^{I K} \subset E^{Q}$ : Assume that $a \notin E^{Q}$. This can be because consumptions are not aligned across states and agents, or because investment levels are outside of $F_{I}^{U}$ (or both). Consider first the former case. Then there is an improvement, $a^{\prime}$, given by the same investment level, and with consumption weights according to some $v \in S^{N}$. Regardless of $q \in \mathcal{Q}_{R}$, and $\lambda \in S^{N}$, it is then the case that $a^{\prime} \succ_{q}^{\lambda} a$, i.e., $a^{\prime}$ strongly IK-dominates $a$, so $a$ is IK-inefficient.

Now consider the case when $I \notin F_{I}^{U}$, but consumption is shared according to some $v \in S^{N}$. W.l.o.g., assume that $I<\min F_{I}^{U}$. Consider now the allocation $a^{\prime}$, which chooses $I^{*}=\min F_{I}^{U}$, with the same consumption weights. Since $U(1-I, I \tilde{R} \mid q)$ is strictly concave in $I$, regardless of $q \in \mathcal{Q}$, and each agent's share is a constant fraction of total consumption in each state, this again immediately implies that $U\left(1-I^{*}, I^{*} \tilde{R} \mid q\right)>U(1-I, \tilde{R} \mid q)$, i.e., $a^{\prime} \succ_{q}^{\lambda} a$ for all $\lambda \in S^{n}$, for all $q \in \mathcal{Q}_{R}$, and therefore again that $a \notin E^{I K}$.

To show that $E^{Q} \subset E^{I K}$, assume that $a \in E^{Q}$, with an associated pair $(q, \lambda)$ (note that there can be multiple $q$ 's, but that $\lambda$ is unique). Obviously, for all $b \in \mathcal{A}, a \succ_{q}^{\lambda} b$, because of uniqueness of the allocations, given Pareto weights and $q$, so indeed $a$ is IK-efficient.

Proof of Proposition 2 Existence:

We follow Basak 2005 (exchange economy) and Baker et al. 2014 (production economy) to use the corresponding planner problem

$$
\begin{aligned}
& U=\max _{I, c^{1}, \ldots, c^{N}} \sum_{n=1}^{N} \hat{\lambda}^{n} U\left(c_{0}^{n}, \tilde{c}_{1}^{n} \mid q^{n}\right), \quad \text { s.t., } \\
& \sum_{n=1}^{N} c_{0}^{n}=K-I, \\
& \sum_{n=1}^{N} c_{1 m}^{n}=R_{m} I, m=1, \ldots, M,
\end{aligned}
$$

to generate equilibria. From the FOC we have

$$
\begin{aligned}
\hat{\lambda}^{n}\left(c_{0}^{n}\right)^{-\gamma} & =\hat{\lambda}^{n^{\prime}}\left(c_{0}^{n^{\prime}}\right)^{-\gamma} \\
\hat{\lambda}^{n} q_{m}^{n}\left(c_{1 m}^{n}\right)^{-\gamma} & =\hat{\lambda}^{n} q_{m}^{n^{\prime}}\left(c_{1 m}^{n^{\prime}}\right)^{-\gamma} .
\end{aligned}
$$


Using the market clearing conditions we can solve for the optimal consumption share of agent $n$

$$
\begin{aligned}
c_{0}^{n} & =\frac{\left(\hat{\lambda}^{n}\right)^{\frac{1}{\gamma}}}{\sum_{n^{\prime}=1}^{N}\left(\hat{\lambda}^{n^{\prime}}\right)^{\frac{1}{\gamma}}}(K-I), \\
c_{1 m}^{n}= & \frac{\left(\hat{\lambda}^{n}\right)^{\frac{1}{\gamma}}\left(q_{m}^{n}\right)^{1 / \gamma}}{\sum_{n^{\prime}=1}^{N}\left(\hat{\lambda}^{n^{\prime}}\right)^{\frac{1}{\gamma}}\left(q_{m}^{n^{\prime}}\right)^{1 / \gamma}} R_{m} I .
\end{aligned}
$$

Rather than working with the Pareto weights, $\hat{\lambda}^{n}$ it is convenient to define, $\lambda^{n}=\left(\hat{\lambda}^{n}\right)^{\frac{1}{\gamma}}$. In addition, define $a_{m n}=\left(q_{m}^{n}\right)^{\frac{1}{\gamma}}$. We can then write the consumption shares as

$$
\begin{aligned}
c_{0}^{n} & =\frac{\lambda^{n}}{\sum_{n^{\prime}}^{N} \lambda^{n^{\prime}}}(K-I), \\
c_{1 m}^{n} & =\frac{\lambda^{n} a_{m n}}{\sum_{n^{\prime}=1}^{N} \lambda_{m}^{n^{\prime}} a_{m n^{\prime}}} R_{m} I .
\end{aligned}
$$

Let $z_{m}=R_{m}^{1-\gamma}, T_{m}=\sum_{n^{\prime}} \lambda^{n^{\prime}} a_{m n^{\prime}}$ and $\alpha(\lambda)=\sum_{m=1}^{M} z_{m} T_{m}^{\gamma}$. Plugging the optimal consumption share into the the social planner's problem, we get

$$
U=\max _{I}\left(\frac{\left(\sum_{n=1}^{N} \lambda^{n}\right)^{\gamma}}{1-\gamma}(1-I)^{1-\gamma}+\alpha(\lambda) \frac{1}{1-\gamma} I^{1-\gamma}\right) .
$$

The first-order condition is

$$
-\left(\sum_{n=1}^{N} \lambda^{n}\right)^{\gamma}(1-I)^{-\gamma}+\alpha(\lambda) I^{-\gamma}=0 .
$$

Solving for the optimal $I$ gives

$$
I=\frac{Z}{\sum_{n=1}^{N} \lambda^{n}+Z},
$$

where $Z$ is the investment to consumption ratio given by

$$
Z=\alpha(\lambda)^{\frac{1}{\gamma}}
$$

We calculate the mapping from the planner's weights, $\lambda$ to initial allocations allocations, $K$. To this end, note that the budget condition for agent $n=1, \ldots, N$ is given by

$$
K^{n}=c_{0}^{n}+E\left[\tilde{\xi}^{n} \tilde{c}_{1}^{n} \mid q^{n}\right]
$$

where $\xi^{n}=\left(\frac{c_{m}^{n}}{c_{0}^{n}}\right)^{-\gamma}$ is the stochastic discount factor. Using the optimal consumption of agent $n$ we can calculate 
the expression in Equation (12) as

$$
\begin{aligned}
K^{n} & =c_{0}^{n}+E\left[\tilde{\xi}^{n} \tilde{c}_{1}^{n} \mid q^{n}\right] \\
& =\frac{\lambda^{n}}{\sum_{n^{\prime}}^{N} \lambda^{n^{\prime}}}(1-I(\lambda))+\sum_{m=1}^{M} q_{m}^{n}\left(\frac{\frac{\lambda^{n} a_{m n}}{\sum_{n^{\prime}=1}^{N} \lambda_{m}^{n^{\prime}} a_{m n^{\prime}}} R_{m} I(\lambda)}{\frac{\lambda^{n}}{\sum_{n^{\prime}}^{N} \lambda^{n^{\prime}}}(1-I(\lambda))}\right)^{-\gamma} \frac{\lambda^{n} a_{m n}}{\sum_{n^{\prime}=1}^{N} \lambda_{m}^{n^{\prime}} a_{m n^{\prime}}} R_{m} I(\lambda) \\
& =\frac{\lambda^{n}}{\sum_{n^{\prime}}^{N} \lambda^{n^{\prime}}}(1-I(\lambda))+I(\lambda) \lambda^{n} \sum_{m=1}^{M} z_{m} a_{m n} T_{m}^{\gamma-1} \\
& =\frac{\lambda^{n}}{\sum_{n^{\prime}} \lambda^{n^{\prime}}}\left(1-I(\lambda)+I(\lambda) F^{n}(\lambda)\right) \\
& =Y^{n}(\lambda)
\end{aligned}
$$

where we have defined $F^{n}(\lambda)=\frac{L}{\alpha} G^{n}(\lambda), G^{n}(\lambda)=\sum_{m} z_{m} a_{m n} T_{m}^{\gamma-1}$ and $L=\sum_{n^{\prime}} \lambda^{n^{\prime}}$. We note that the function $Y(\lambda)=\left(Y^{1}(\lambda), \ldots, Y^{N}(\lambda)\right)^{\prime}$ is defined on the whole of $\bar{S}^{N}$.

Because of the functional mapping $K=Y(\lambda)$, it is easy to show that the mapping $K=Y(\lambda)$ is surjective, that is, that for every $K$, there exists a $\lambda$ such that $K=Y(\lambda)$. Note that $Y(\lambda)=\frac{\lambda^{n}}{\sum_{n^{\prime}} \lambda^{n^{\prime}}}\left(1-I(\lambda)+I(\lambda) F^{n}(\lambda)\right)$ is homogeneous of degree zero. Hence, we can without loss of generality focus on $\lambda$ on the unit simplex, $\bar{S}^{N}$, which is a compact and convex set. It follow immediately that $Y$ is continuous on $\bar{S}^{N}$, being a product of continuous functions on this domain.

Define the function $f: \bar{S}^{N} \rightarrow \bar{S}^{N}$, by $f(\lambda)=Y(\lambda)-K+\lambda$. Here, $K$ is treated as a constant parameter. That $f(\lambda)$ maps the unit simplex into itself follows from the fact that $\sum_{n} Y^{n}(\lambda)=1$, and $\sum_{n} K^{n}=1$. Since $f$ is continuous, and $\bar{S}^{N}$ is compact and convex, Brouwer's fixed theorem point now immediately implies that $f$ has a fixed point, $\lambda^{*}$, and it therefore follows that $Y\left(\lambda^{*}\right)=K$.

Thus, $Y$ is a surjective mapping from Pareto weights to capital on the unit simplex, associated with the resulting equilibrium state prices $(\mathrm{A})$ :

$$
p_{m}=E\left[\tilde{\xi}^{n} \delta_{m} \mid q^{n}\right]=\left(\frac{c_{m}^{n}}{c_{0}^{n}}\right)^{-\gamma} q_{m}^{n}, \quad m=1, \ldots, M
$$

where $\delta_{m}$ is the random variable, such that $\delta_{m}\left(\omega_{w}\right)=1$, and $\delta_{m}\left(\omega_{m^{\prime}}\right)=0, m^{\prime} \neq m$, representing the payout of the $m$ :th AD security, and any $n=1, \ldots, N$ can be chosen. We have shown the first part of the theorem.

Uniqueness: We first study the case $\gamma \geq 1$. Define

$$
\begin{aligned}
G^{n j} & =\sum_{m} z_{m} a_{m n} a_{m j} T_{m}^{\gamma-2}, \\
F^{n j} & =\frac{L^{2}}{\alpha} G^{n j} .
\end{aligned}
$$

The mapping from Pareto weights to capital, $K=Y(\lambda)$, is given in Equation (13). The partial derivatives are

$$
\begin{aligned}
\frac{\partial I}{\partial \lambda^{j}} & =\frac{1}{\sum_{n^{\prime}} \lambda^{n^{\prime}}} I(1-I)\left(F^{j}-1\right) \\
\frac{\partial F}{\partial \lambda^{j}} & =\frac{1}{\sum_{n^{\prime}} \lambda^{n^{\prime}}}\left(F^{n}+(\gamma-1) F^{n j}-\gamma F^{n} F^{j}\right) .
\end{aligned}
$$

We define the matrix $Q$, with elements

$$
[Q]_{n j}=-(1-I)^{2}-I(1-I)\left(F^{n}+F^{j}\right)-(\gamma-1) I F^{n j}-(\gamma+I-1) I F^{n} F^{j}, \quad 1 \leq n, j \leq N,
$$

and then get

$$
\frac{\partial Y^{n}}{\partial \lambda^{j}}=\frac{\lambda^{n}}{L^{2}} Q_{n j}, \quad j \neq n .
$$


Moreover, we get

$$
\frac{\partial Y^{n}}{\partial \lambda^{n}}=\frac{1}{L}\left(1-I+I F^{n}\right)+\frac{\lambda^{n}}{L^{2}} Q_{n n}
$$

We next do a change of coordinate transformation to $y$, where $y_{n}=\log \left(\lambda^{n}\right)$, i.e., $\lambda^{n}=e^{y_{n}}$, i.e., we write

$$
K^{n}=\hat{Y}(y)=\frac{e^{y_{n}}}{L}\left(1-I\left(e^{y}\right)+I\left(e^{y}\right) F^{n}\left(e^{y}\right)\right)
$$

It follows from the chain rule that partial derivatives w.r.t. $y$ will be similar as w.r.t. $\lambda$, but with extra $e^{y_{j}}$ inner derivative terms:

$$
\begin{aligned}
\frac{\partial \hat{Y}^{n}}{\partial y_{j}} & =\frac{e^{y_{n}} e^{y_{j}}}{L^{2}} Q_{n j}, \quad j \neq n \\
\frac{\partial \hat{Y}^{n}}{\partial y_{n}} & =\frac{e^{y_{n}}}{L}\left(1-I+I F^{n}\right)+\frac{e^{y_{n}} e^{y_{n}}}{L^{2}} Q_{n n}
\end{aligned}
$$

Now we rewrite this on matrix form, defining the matrix $H\left(e^{y}\right)$, with elements $[H]_{n j}=\frac{\partial \hat{Y}^{n}}{\partial y_{j}}$, to get:

$$
H(\lambda)=\frac{1}{L^{2}} \Lambda_{\lambda} Q \Lambda_{\lambda}+\frac{1}{L} \Lambda_{\lambda}^{1 / 2} \Lambda_{w} \Lambda_{\lambda}^{1 / 2}
$$

Here, for a general vector, $x$, we use the notation $\Lambda_{v}=\operatorname{diag}(x)$, and we define the vector

$$
w=(1-I) \mathbf{1}+I F,
$$

treating $F$ as a vector. Moreover,

$$
\begin{aligned}
Q & =-(1-I)^{2} \mathbf{1} \mathbf{1}^{\prime}-I(1-I)\left(F \mathbf{1}^{\prime}+\mathbf{1} F^{\prime}\right)+(\gamma-1) I R-(\gamma+I-1) I F F^{\prime} \\
& =-\left((1-I)^{2} \mathbf{1 1}^{\prime}+I(1-I)\left(F \mathbf{1}^{\prime}+\mathbf{1} F^{\prime}\right)+I^{2} F^{\prime}\right)+(\gamma-1) I\left(R-F F^{\prime}\right) \\
& =-w w^{\prime}+(\gamma-1) I\left(R-F F^{\prime}\right),
\end{aligned}
$$

so we have

$$
H(\lambda)=\underbrace{\frac{1}{L} \Lambda_{\lambda}^{1 / 2} \Lambda_{w} \Lambda_{\lambda}^{1 / 2}-\frac{1}{L^{2}} \Lambda_{\lambda}\left(w w^{\prime}\right) \Lambda_{\lambda}}_{C}+(\gamma-1) I \frac{1}{L^{2}} \Lambda_{\lambda}\left(R-F F^{\prime}\right) \Lambda_{\lambda} .
$$

Here, the matrix $R$ is the matrix with elements $[R]_{n j}=F^{n j}$, and we note that by defining the matrix $a \in \mathbb{R}_{+}^{M \times N}$ with elements $a_{m n}$, it can be written on self adjoint matrix form as $R=\frac{L^{2}}{\alpha} a^{\prime} \Lambda_{v} a$, where $v_{m}=T_{m}^{\gamma-2} z_{m}$. Thus, $R$ is positive semidefinite. Note that homogeneity of $Y$ implies that

$$
H(\lambda) \mathbf{1}=\mathbf{0}
$$

We have $F=\frac{L}{\alpha} a^{\prime} w$, where $w_{m}=T^{\gamma-1} z_{m}$, and thus $F F^{\prime}=\frac{L^{2}}{\alpha^{2}} a^{\prime} w w^{\prime} a$, leading to

$$
\begin{aligned}
R-F F^{\prime} & =\frac{L^{2}}{\alpha} a^{\prime}\left(\Lambda_{v}-\frac{1}{\alpha} w w^{\prime}\right) a \\
& =\frac{L^{2}}{\alpha} a^{\prime} \Lambda_{v}^{1 / 2}\left(E-\frac{1}{\alpha} \Lambda_{v}^{-1 / 2} w w^{\prime} \Lambda_{v}^{-1 / 2}\right) \Lambda_{v}^{1 / 2} a
\end{aligned}
$$

Note that $\Lambda_{v}^{-1 / 2} w=\left(T_{1}^{\gamma / 2} z_{1}^{1 / 2}, \ldots, T_{M}^{1 / 2} z_{m}^{1 / 2}\right)$. Also, recall that the eigenvalues of the 1-rank perturbation of the identity matrix $E-x x^{\prime}$ are 1 with multiplicity $N-1$, and $1-\left(x^{\prime} x\right)$ with multiplicity 1 , where $x$ is the vector 
with eigenvalue $1-\left(x^{\prime} x\right)$. Now, $\left(\Lambda_{v}^{-1 / 2} w\right)^{\prime}\left(\Lambda_{v}^{-1 / 2} w\right)=\sum_{m}\left(T_{m}^{\gamma / 2} z_{m}^{1 / 2}\right)^{2}=\alpha$, and thus the eigenvalues of $E-$ $\frac{1}{\alpha} \Lambda_{v}^{-1 / 2} w w^{\prime} \Lambda_{v}^{-1 / 2}$ are 1 with multiplicity $N-1$, and 0 with multiplicity 1 . So, Sylvester's law of inertia (on general rectangular form, see Higham and Cheng (1998)), implies that $R-F F^{\prime}$ is also positive semidefinite, with number of 0 eigenvalues dependent on $N, M$, and the rank of $a$.

It is easy to verify that $R \Lambda_{\lambda} \mathbf{1}=R \lambda=L F$, which in turn implies that the generic zero eigenvalue is generated by $\mathbf{1}$, since $F F^{\prime} \Lambda_{\lambda} \mathbf{1}=F F^{\prime} \lambda=F L$, so

$$
\left(R-F F^{\prime}\right) \Lambda_{\lambda} \mathbf{1}=\mathbf{0}
$$

Next, consider the remaining term

$$
\begin{aligned}
C & =\frac{1}{L} \Lambda_{\lambda}^{1 / 2} \Lambda_{w} \Lambda_{\lambda}^{1 / 2}-\frac{1}{L^{2}} \Lambda_{\lambda} w w^{\prime} \Lambda_{\lambda} \\
& =\frac{1}{\sqrt{L}} \Lambda_{\lambda}^{1 / 2} \Lambda_{w}^{1 / 2}\left(E-g g^{\prime}\right) \Lambda_{w}^{1 / 2} \Lambda_{\lambda}^{1 / 2} \frac{1}{\sqrt{L}}
\end{aligned}
$$

where

$$
g_{n}=\sqrt{\frac{\lambda_{n} w_{n}}{L}}
$$

It immediately follows that $g^{\prime} g=\frac{1}{L} \sum_{n} \lambda_{n}\left((1-I)+I F_{n}\right)=1$, and therefore a similar argument as for $R-F F^{\prime}$ implies that there is one eigenvalue of $C$ which is 0 , and all the other eigenvalues are 1 . It is also easy to check that $\mathbf{1}$ is the eigenvector that corresponds to the eigenvalue 0 .

Thus, altogether, since $q^{\prime}(A+B) q=q^{\prime} A q+q^{\prime} B q$ for general matrices $A$ and $B$, it follows that $H$ is positive semidefinite, with exactly one zero eigenvalue and the corresponding eigenvector $\mathbf{1}$, for all $\lambda$.

Now, assume that $Y\left(\lambda_{1}\right)=Y\left(\lambda_{2}\right)$ for distinct $\lambda_{1}$ and $\lambda_{2}$ (i.e., such that it is not the case that $\lambda_{2}$ is proportional to $\left.\lambda_{1}\right)$. Define $y_{1}=\log \left(\lambda_{1}\right)$ and $y_{2}=\log \left(\lambda_{2}\right)$. It then follows that $\hat{Y}\left(y_{2}\right)-\hat{Y}\left(y_{1}\right)=\mathbf{0}$, and thus that $\left(y_{2}-y_{1}\right)^{\prime}\left(\hat{Y}\left(y_{2}\right)-\right.$ $\left.\hat{Y}\left(y_{1}\right)\right)=0$. Define $\Delta y=y_{2}-y_{1}$, to get

$$
\hat{Y}\left(y_{2}\right)-\hat{Y}\left(y_{1}\right)=\int_{s=0}^{s=1} H\left(e^{y_{1}+s \Delta y}\right)(\Delta y) d s=0
$$

and therefore

$$
\int_{s=0}^{s=1}(\Delta y)^{\prime} H\left(e^{y_{1}+s \Delta y}\right)(\Delta y) d s=0 .
$$

However, since $H$ is positive semidefinite for all $y$, with eigenvector $\mathbf{1}$, this is only possible if $y_{2}-y_{1}=c \mathbf{1}$, for some constant $c$, i.e., only in the proportional case, $\frac{\lambda_{2}^{n}}{\lambda_{n}^{1}}=e^{c}$ for all $n$.

For $\gamma<1$, it follows immediately from the definition of $Q$ that as long as $\gamma-1+I>0, \partial Y^{n} / \partial \lambda^{j}<0$, which means that the Gross Substitution property holds, and thereby that equilibrium is unique. Since $I>0$, there is thus always a $\underline{\gamma}<1$, such that this property holds for all $\gamma>\underline{\gamma}$. This completes the second part of the Proposition.

Proof of Proposition 3 In an economy with homogeneous beliefs $q$, it follows from the first welfare theorem that any equilibrium allocation, $a \in E_{q}$, and by Proposition 1, the allocation is therefore IK-efficient.

In the heterogeneous beliefs economy, $q_{m}^{n}>q_{m}^{n^{\prime}}$ and $q_{m^{\prime}}^{n}<q_{m^{\prime}}^{n^{\prime}}$ for some $n, n^{\prime}, m, m^{\prime}$, and the equilibrium condition:

$$
\frac{1}{v^{n}} c_{1 m}^{n}\left(q_{m}^{n}\right)^{1 / \gamma}=\frac{1}{v^{n^{\prime}}} c_{1 m}^{n^{\prime}}\left(q_{m}^{n^{\prime}}\right)^{1 / \gamma}, \quad m=1, \ldots, M
$$

implies that consumption shares are not constant across states. Therefore, the allocation does not belong to $E_{q}$ for any $q$. Again using Proposition 1, it follows that the equilibrium allocation is IK-inefficient.

Proof of Proposition 4 The only unanimously reasonable value for the speculative component, $s$, is zero. Hence, we need to show that any economy with disagreement will have a non-zero speculative component. This follows directly from the proof of Proposition 3 as the consumption shares are not constant across states. 
Proof of Proposition 5 To show this is it sufficient to look at the optimal investment for all reasonable beliefs. Agents agree on aggregate risk, hence we have that $E\left[\tilde{R}^{1-\gamma} \mid q^{n}\right]=E\left[\tilde{R}^{1-\gamma} \mid q^{n^{\prime}}\right]$ for all $q^{n}, q^{n^{\prime}} \in \mathcal{Q}_{R}$. From the FOC we have that the optimal investment is

$$
I^{*}=\frac{Z}{1+Z}
$$

where $Z=\left(E\left[\tilde{R}^{1-\gamma} \mid q\right]\right)^{\frac{1}{\gamma}}$. As $E\left[\tilde{R}^{1-\gamma} \mid q\right]$ is the same for all $q \in \mathcal{Q}_{R}$, the set of reasonable investments contains the single element $I^{*}$.

Proof of Proposition 6 The equilibrium investment-to-consumption ratio, $Z$, is given by

$$
Z=\left(\sum_{m=1}^{M} R_{m}^{1-\gamma}\left(\sum_{n=1}^{N} \lambda^{n}\left(q_{m}^{n}\right)^{\frac{1}{\gamma}}\right)^{\gamma}\right)^{\frac{1}{\gamma}}
$$

By Jensen's inequality we have that

$$
\begin{aligned}
& Z<\left(\sum_{m=1}^{M} R_{m}^{1-\gamma}\left(\sum_{n=1}^{N} \lambda^{n}\left(q_{m}^{n}\right)\right)\right)^{\frac{1}{\gamma}}, \quad \gamma<1 \\
& Z=\left(\sum_{m=1}^{M} R_{m}^{1-\gamma}\left(\sum_{n=1}^{N} \lambda^{n}\left(q_{m}^{n}\right)\right)\right)^{\frac{1}{\gamma}}, \quad \gamma=1 \\
& Z>\left(\sum_{m=1}^{M} R_{m}^{1-\gamma}\left(\sum_{n=1}^{N} \lambda^{n}\left(q_{m}^{n}\right)\right)\right)^{\frac{1}{\gamma}}, \quad \gamma>1 .
\end{aligned}
$$

As agents agree on aggregate risk we have that $\left(\sum_{m=1}^{M} R_{m}^{1-\gamma}\left(\sum_{n=1}^{N} \lambda^{n}\left(q_{m}^{n}\right)\right)\right)^{\frac{1}{\gamma}}=\left(E\left[\tilde{R}^{1-\gamma} \mid q^{n}\right]\right)^{\frac{1}{\gamma}}$ for all $n=$ $1, \ldots, N$. Hence, the only case in which the aggregate investment is equal to the unanimously reasonable investments is when agents have log utility.

Proof of Proposition 7 This follows directly from the proof of Proposition 6 .

Proof of Proposition 8 The result follows from the first welfare theorem. Specifically, as discussed in the proof of Proposition 2, the equilibrium outcome is the Pareto optimal solution to the social planner's problem for a representative agent model with an agent with state dependent utility that can be written as a weighted sum (corresponding to the Pareto weights) of the individual agents' utilities. Moreover, the solution is unique, given the specific Pareto weights, as follows from $(9,10)$.

Now, if there is an allocation, $b \neq a$, that U-dominates an equilibrium outcome, $a$, it must weakly dominate $a$ for all $q \in \mathcal{Q}_{R}$, and thus for each agent when using their individual beliefs, $q^{n} \in \mathcal{Q}_{R}$. But this means that $b$ is another solution to the social planner's problem, contradicting the uniqueness of such solutions. So, no such alternative $b$ exists, and $a$ is thus U-efficient. We are done.

\section{Proof of Proposition 9}

First, to prove that there is never intratemporal mispricing we need to calculate the relative AD prices in autarky equilibrium for each agent. Consider agent $n$. The relative AD price of state $m$ and $m^{\prime}, p_{m, m^{\prime}}^{n}=\frac{p_{m}^{n}}{p_{m}^{n}}$, is

$$
p_{m, m^{\prime}}^{n}=\left(\frac{q_{m}^{n}}{q_{m^{\prime}}^{n}}\right)\left(\frac{R_{m}}{R_{m^{\prime}}}\right)^{-\gamma}
$$

Hence, the set of reasonable relative AD prices are $\left[\min _{n}\left(\frac{q_{m}^{n}}{q_{m^{\prime}}^{n}}\right)\left(\frac{R_{m}}{R_{m^{\prime}}}\right)^{-\gamma}, \max _{n}\left(\frac{q_{m}^{n}}{q_{m^{\prime}}^{n}}\right)\left(\frac{R_{m}}{R_{m^{\prime}}}\right)^{-\gamma}\right]$. Next, we calculate 
the equilibrium $\mathrm{AD}$ ratios, $p_{m, m^{\prime}}$. As agents agree on the prices, it is sufficient to use the marginal utility of any agent in the economy. Hence, we have

$$
p_{m, m^{\prime}}=\left(\frac{q_{m}^{n}}{q_{m^{\prime}}^{n}}\right)\left(\frac{R_{m}}{R_{m^{\prime}}}\right)^{-\gamma}\left(\frac{f_{m}^{n}}{f_{m^{\prime}}^{n}}\right)^{-\gamma} .
$$

Therefore, if we can show that for agent $\bar{n}=\underset{n}{\arg \max } p_{m, m^{\prime}}^{n}$ that $\frac{f_{m}^{\bar{n}}}{f_{m^{\prime}}^{n}} \geq 1$ and for agent $\underline{n}=\underset{n}{\arg \min } p_{m, m^{\prime}}^{n}$ that $\frac{f \frac{n}{m}}{f_{m^{\prime}}^{n}} \leq 1$ we are done. Note that

$$
\frac{f_{m}^{\bar{n}}}{f_{m^{\prime}}^{\bar{n}}}=\left(\frac{\sum_{n} \lambda^{n}\left(q_{m^{\prime}}^{n}\right)^{\frac{1}{\gamma}}}{\sum_{n} \lambda^{n}\left(q_{m}^{n}\right)^{\frac{1}{\gamma}}}\right)\left(\frac{q_{m}^{\bar{n}}}{q_{m^{\prime}}^{\bar{n}}}\right) .
$$

Let $G_{m}=\sum_{n} \lambda^{n} a_{m}^{n}$ where $a_{m}^{n}=\left(q_{m}^{n}\right)^{\frac{1}{\gamma}}$. Hence, we can write $\frac{f_{m}^{\bar{n}}}{f_{m^{\prime}}^{n}}=\left(\frac{G_{m}^{\prime}}{G_{m}}\right)\left(\frac{a_{m}^{n}}{a_{m^{\prime}}^{n}}\right)$. We have $\frac{f_{m}^{\bar{n}}}{f_{m^{\prime}}^{\bar{n}}} \geq 1 \Leftrightarrow \frac{G_{m}}{G_{m^{\prime}}} \geq \frac{a_{m}^{\bar{n}}}{a_{m^{\prime}}^{n}}$. We will show that $\frac{G_{m}}{G_{m^{\prime}}}>\frac{a_{m}^{\bar{n}}}{a_{m^{\prime}}^{n}}$ cannot be the case by contradiction. Note that $\frac{G_{m}}{G_{m^{\prime}}}>\frac{a_{m}^{\bar{n}}}{a_{m^{\prime}}^{n}} \Leftrightarrow G_{m}>\frac{a_{m}^{\bar{n}}}{a_{m^{\prime}}^{n}} G_{m^{\prime}}$. We can write this as

$$
\begin{aligned}
G_{m} & >\frac{a_{m}^{\bar{n}}}{a_{m^{\prime}}^{\bar{n}}} G_{m^{\prime}} \\
\sum_{n} \lambda^{n} \frac{a_{m}^{n}}{a_{m^{\prime}}^{n}} a_{m^{\prime}}^{n} & >\frac{a_{m}^{\bar{n}}}{a_{m^{\prime}}^{\bar{n}}} G_{m^{\prime}}
\end{aligned}
$$

However, $\sum_{n} \lambda^{n} \frac{a_{m}^{n}}{a_{m^{\prime}}^{n}} a_{m^{\prime}}^{n} \leq \frac{a_{m}^{\bar{n}}}{a_{m^{\prime}}^{n}} \sum_{n} \lambda^{n} a_{m^{\prime}}^{n}$ which proves that $\frac{G_{m}}{G_{m^{\prime}}} \leq \frac{a_{m}^{\bar{n}}}{a_{m^{\prime}}^{n}}$. The proof for $\frac{f_{m}^{n}}{f_{m^{\prime}}^{n}} \leq 1$ is similar.

Proof of Proposition 10: Define $z_{m}=R_{m}^{-\gamma}, m=1, \ldots, M$. In homogeneous beliefs equilibrium for agent $n$, we have:

$$
\frac{z_{m}}{z_{m^{\prime}}} \frac{q_{m}^{n}}{q_{m^{\prime}}^{n}}=\frac{p_{m}^{n}}{p_{m^{\prime}}^{n}}, \quad 1 \leq m, m^{\prime} \leq M, 1 \leq n \leq N
$$

Consider portfolios $w$ and $v$. Agent $n$ 's value ratio for these two portfolios is:

$$
\frac{\sum_{m} w_{m} p_{n}^{m}}{\sum_{m} v_{m} p_{m}^{n}}=\frac{\sum_{m} w_{m} z_{m} q_{m}^{n} p_{1}^{n} /\left(z_{1} q_{1}^{n}\right)}{\sum_{m} v_{m} z_{m} q_{m}^{n} p_{1}^{n} /\left(z_{1} q_{1}^{n}\right)}=\frac{\sum_{m} w_{m} z_{m} q_{m}^{n}}{\sum_{m} v_{m} z_{m} q_{m}^{n}} .
$$

If $\sum_{m} w_{m} z_{m} q_{m}^{n}=0, n=1, \ldots, N$, all agents therefore agree that the price of asset $w$ should be 0 . In matrix form, defining the matrix $\Pi$ with $\Pi_{m n}=q_{m}^{n}=\left(A_{m n}\right)^{\gamma}$, we write this as

$$
\Pi \Lambda_{z} w=\mathbf{0}
$$

Now, since $N<M$, it is clear that a nontrivial such asset with unanimous price 0 exists. For convenience, we define $r=\Lambda_{z} w$, and we then have $\Pi r=\mathbf{0}$. Note that every agent with beliefs in $\mathcal{Q}_{R}$ also agrees on the reasonable price being zero, since

$$
\begin{aligned}
\sum_{m} w_{m} z_{m}\left(\lambda q_{m}^{n_{1}}+(1-\lambda) q_{m}^{n_{2}}\right) & =\lambda \sum_{m} w_{m} z_{m} q_{m}^{n_{1}}+(1-\lambda) \sum_{m} w_{m} z_{m} q_{m}^{n_{2}} \\
& =\lambda \times 0+(1-\lambda) \times 0 \\
& =0 .
\end{aligned}
$$

The equilibrium price of $w$ under Pareto weights $\lambda \in S^{n}$, in terms of time 0 consumption, is

$$
\frac{\sum_{m}\left(\sum_{n} A_{m n} \lambda^{n}\right)^{\gamma} r_{m}}{\sum_{m}\left(\sum_{n} A_{m n} \lambda^{n}\right)^{\gamma} \tilde{R}^{1-\gamma}}
$$


so there is intertemporal mispricing of this asset if and only if

$$
Q(\lambda)=\sum_{m}\left(\sum_{n} A_{m n} \lambda^{n}\right)^{\gamma} r_{m} \neq 0
$$

We wish to show that under the conditions of the proposition, (22) is typically satisfied except for possibly a small set of $\lambda$ 's. In other words, if we define the set $X=\left\{\lambda \in S^{N}: Q(\lambda)=0\right\}$, we want to show that the Lebesgue measure of $X$ is zero, $\mu_{S^{N}}(X)=0$. Of course, since $Q$ is a continuous function, and $X=Q^{-1}(\{0\})$ is the preimage of a Borel set, $X$ is measurable.

We use the following standard lemma:

Lemma 1 Consider the function $f(x)=\sum_{m=1}^{M}\left(1+c_{m} x\right)^{\gamma} b_{m}$, where $\gamma>0, \gamma \neq 1, c_{m}>-1, b_{m} \in \mathbb{R}, m=1, \ldots, M$. If there are at least two distinct $c^{\prime} s, c_{m} \neq c_{m^{\prime}}$, the number of roots of $f(x)$ in $x \in[0,1]$ is finite.

Proof: The proof follows from the Principle of Permanence, given the facts that $f$ is a real analytic function on the interval $x \in[-\epsilon, 1+\epsilon]$ for some $\epsilon>0$, and that $f$ is not a constant function as long as $c_{m} \neq c_{m^{\prime}}$.

We use Lemma 1 to show that on any ray between two distinct Pareto weights, $\lambda_{a}, \lambda_{b} \in S^{N}, Q(\lambda)$ is nonzero, except possibly at a finite number of points. Indeed, consider the function

$$
\begin{aligned}
g(x) & =\sum_{m}\left(x \sum_{n} A_{m n} \lambda_{a}+(1-x) \sum_{n} A_{m n} \lambda_{b}\right)^{\gamma} r_{m} \\
& =\sum_{m}\left(\sum_{n} A_{m n} \lambda_{b}^{n}+x\left(\sum_{n} A_{m n} \lambda_{a}^{n}-\sum_{n} A_{m n} \lambda_{b}^{n}\right)\right)^{\gamma} r_{m} \\
& =\sum_{m}\left(1+x \frac{\sum_{n} A_{m n} \lambda_{a}^{n}-\sum_{n} A_{m n} \lambda_{b}^{n}}{\sum_{n} A_{m n} \lambda_{b}^{n}}\right)^{\gamma}\left(\sum_{n} A_{m n} \lambda_{b}^{n}\right)^{\gamma} r_{m} \\
& =\sum_{m}\left(1+c_{m} x\right)^{\gamma} b_{m} .
\end{aligned}
$$

For all $c_{m}$ 's to be the same, it must be that $A \lambda_{a}=k A \lambda_{b}$, but since the rank of $A$ is $N$, this implies that $\lambda_{a}=k \lambda_{b}$, and given that both $\lambda_{a}$ and $\lambda_{b}$ are in $S^{n}$, that $\lambda_{a}=\lambda_{b}$, contradicting our assumption that the two vectors are distinct. Thus, Lemma 1 implies that there is a finite number of roots of (22) on any such line.

That $\mu_{S^{N}}(X)=0$ now follows immediately from Fubini's theorem. Specifically, consider the characteristic function on $X, \chi_{X}$. Any line integral of $\chi_{X}$ is zero, $\int_{\lambda_{1}} \chi_{X}(\lambda) d \lambda=0$, since $X$ only contains a finite number of points along any line. Fubini's theorem then implies that the total Lebesgue integral can be viewed as an $N-2$-dimensional integral over such line integrals, each of which integrates to zero, and therefore that

$$
\int_{S^{N}} \chi_{X}(\lambda) d \lambda=\int_{\lambda_{2}, \ldots, \lambda_{N-1}}\left(\int_{\lambda_{1}} \chi_{X}(\lambda) d \lambda_{1}\right) d \lambda_{2} \cdots d \lambda_{N-1}=0
$$

We are done.

Proof of Proposition 11: The unanimously reasonable relative price $\frac{p_{m}}{p_{B}}$, where $p_{B}=\sum_{m^{\prime} \in B} p_{m^{\prime}}$ is $\mathbb{P}\left(\omega_{m} \mid B\right)=$ 
$\frac{q_{m}^{n}}{\sum_{m^{\prime} \in B} q_{m^{\prime}}^{n}}=\frac{q_{m}^{n^{\prime}}}{\sum_{m^{\prime} \in B} q_{m^{\prime}}^{n^{\prime}}}$ for all $n, n^{\prime}=1, \ldots, N$. The equilibrium price is

$$
\begin{aligned}
\frac{p_{m}}{p_{B}} & =\frac{\left(\sum_{n=1}^{N} \lambda^{n}\left(q_{m}^{n}\right)^{\frac{1}{\gamma}}\right)^{\gamma}}{\sum_{m^{\prime} \in B}\left(\sum_{n=1}^{N} \lambda^{n}\left(q_{m^{\prime}}^{n}\right)^{\frac{1}{\gamma}}\right)^{\gamma}} \\
& =\frac{\left(\sum_{n=1}^{N} \lambda^{n}\left(q_{m}^{n}\right)^{\frac{1}{\gamma}}\right)^{\gamma}}{\sum_{m^{\prime} \in B}\left(\sum_{n=1}^{N} \lambda^{n}\left(\frac{q_{m^{\prime}}^{n}}{q_{m}^{n}}\right)^{\frac{1}{\gamma}}\left(q_{m}^{n}\right)^{\frac{1}{\gamma}}\right)^{\gamma}} \\
& =\frac{1}{\sum_{m^{\prime} \in B}\left(\sum_{n=1}^{N} \frac{\lambda^{n}\left(q_{m}^{n}\right)^{\frac{1}{\gamma}}}{\left(\sum_{n=1}^{N} \lambda^{n}\left(q_{m}^{n}\right)^{\frac{1}{\gamma}}\right)^{\gamma}}\left(\frac{q_{m^{\prime}}^{n}}{q_{m}^{n}}\right)^{\frac{1}{\gamma}}\right)^{\gamma}} \\
& =\frac{1}{\sum_{m^{\prime} \in B}\left(\sum_{n=1}^{N} W_{n}\left(\frac{q_{m^{\prime}}^{n}}{q_{m}^{n}}\right)^{\frac{1}{\gamma}}\right)^{\gamma}}
\end{aligned}
$$

where $\sum_{n=1}^{N} W_{n}=1$. Note that the only reasonable price can be written as $\frac{1}{\sum_{m^{\prime} \in B} \frac{q_{m}^{n} q_{m}^{\prime}}{q_{m}^{n}}}$. Hence, only if $\sum_{m^{\prime} \in B} \frac{q_{m}^{n}}{q_{m}^{n}}=$ $\sum_{m^{\prime} \in B}\left(\sum_{n=1}^{N} W_{n}\left(\frac{q_{m^{\prime}}^{n}}{q_{m}^{n}}\right)^{\frac{1}{\gamma}}\right)^{\gamma}$ will there be no mispricing. Define $H=\sum_{m^{\prime} \in B}\left(\sum_{n=1}^{N} W_{n}\left(\frac{q_{m^{\prime}}^{n}}{q_{m}^{n}}\right)^{\frac{1}{\gamma}}\right)^{\gamma}$. By Jensen's inequality we have

$$
\begin{aligned}
& H<\sum_{m^{\prime} \in B}\left(\sum_{n=1}^{N} W_{n}\left(\frac{q_{m^{\prime}}^{n}}{q_{m}^{n}}\right)\right)=\sum_{m^{\prime} \in B} \frac{q_{m^{\prime}}^{n}}{q_{m}^{n}}, \quad \gamma<1 \\
& H=\sum_{m^{\prime} \in B}\left(\sum_{n=1}^{N} W_{n}\left(\frac{q_{m^{\prime}}^{n}}{q_{m}^{n}}\right)\right)=\sum_{m^{\prime} \in B} \frac{q_{m^{\prime}}^{n}}{q_{m}^{n}}, \quad \gamma=1 \\
& H>\sum_{m^{\prime} \in B}\left(\sum_{n=1}^{N} W_{n}\left(\frac{q_{m^{\prime}}^{n}}{q_{m}^{n}}\right)\right)=\sum_{m^{\prime} \in B} \frac{q_{m^{\prime}}^{n}}{q_{m}^{n}}, \quad \gamma>1 .
\end{aligned}
$$

Hence, as long as $\gamma \neq 1$, which we rule out by assumption, there will be mispricing.

Proof of Proposition 12:

The equilibrium outcome in the economy without an investment tax is a solution to the planner's problem:

$$
\max _{I, g_{0}^{n}, g_{1 m}^{n}} \sum_{n} \lambda^{n}\left((1-I)^{1-\gamma} \frac{\left(g_{0}^{n}\right)^{1-\gamma}}{1-\gamma}+I^{1-\gamma} \sum_{m} \frac{\left(g_{1 m}^{n}\right)^{1-\gamma} z_{m} q_{m}^{n}}{1-\gamma}\right)
$$

where $g_{m}^{n}$ represents the consumption share of agent $n$ in state $m$, and the $g$ 's sum to unity across agents in each state, and $z_{m}=R_{m}^{1-\gamma}$. The Pareto weights, $\lambda$ are chosen to match agents' initial allocations, $K$. In the case we are investigating, $\psi=\frac{1}{\gamma}<1$, there is underinvestment in equilibrium, $I<I^{*}$. Denote this equilibrium allocation by $a$.

Now, consider the case where an investment tax is introduced. For every unit invested, the government collects $\tau$, so from each agent's perspective, an investment of $I$ at $t=0$ then results in $(1-\tau) I \tilde{R}$ at $t=1$. The tax is constructed to be revenue neutral: the $\tau I$ raised by the tax is distributed back to the agents at $t=0$ (and thus not invested), in proportion so as to keep the Pareto weights in the social planner problem constant, leading to an outcome that solves:

$$
\max _{I, f_{0}^{n}, f_{1 m}^{n}} \sum_{n} \lambda^{n}\left((1+\tau \hat{I}-(1+\tau) I)^{1-\gamma} \frac{\left(f_{0}^{n}\right)^{1-\gamma}}{1-\gamma}+I^{1-\gamma} \sum_{m} \frac{\left(f_{1 m}^{n}\right)^{1-\gamma} z_{m} q_{m}^{n}}{1-\gamma}\right),
$$


where the $f$ 's represent the consumption shares. Here, given $\tau, \hat{I}$ is chosen such that $I=\hat{I}$ in equilibrium (revenue neutrality), leading to real (taxed) investments $I=(1-\tau) \hat{I}$ (since $\tau \hat{I}$ is returned to agents). We therefore view investments as a function $I(\tau)$. To determine the sign of $\frac{d I}{d \tau}$, we note that $I=(1+\tau I) \frac{\hat{Z}}{1+\hat{Z}}$, where $\hat{Z}=(1+\tau)^{1-1 / \gamma} Z$, and $Z$ is defined in (11). The $(1+\tau I)$ term arises in this expression, because the total wealth in the economy is (artificially) viewed as higher than unity in the social planner's problem, because of the lump-sum payment. It is straightforward to show that this leads to

$$
I=\frac{1}{1+\frac{(1+\tau)^{1 / \gamma-1}}{Z}-\tau}
$$

and the partial derivative of the denominator is $\left(\frac{1}{\gamma}-1\right) \frac{(1+\tau)^{1 / \gamma-2}}{Z}-1<0$, leading to $\frac{d I}{d \tau}>0$. Thus, by choosing a positive $\tau$, the equilibrium underinvestment level can be decreased. We also note that since the Pareto weights are the same in the two problems $(23,24)$, the optimal consumption shares are identical, $f_{t m}^{n}=g_{t m}^{n}$, for all $n, t$, and $m$.

We denote the allocation when investment level $\bar{I}$ is chosen by $b_{\bar{I}}$, and for general $\bar{I} \in(I(0), 1], q \in \mathcal{Q}_{R}$, define

$$
U(\bar{I}, q)=\sum_{n} \lambda^{n}\left((1-\bar{I})^{1-\gamma} \frac{\left(f_{0}^{n}\right)^{1-\gamma}}{1-\gamma}+\bar{I}^{1-\gamma} \sum_{m} \frac{\left(f_{1 m}^{n}\right)^{1-\gamma} z_{m} q_{m}}{1-\gamma}\right)
$$

Note that $U(\bar{I}, q)$ does not depend on $q \in \mathcal{Q}_{R}$, since agents agree on aggregate risk. By the definition of weak IK-dominance, if an $I_{0} \in(I(0), 1]$ (and associated tax level, $\tau$ ) exists, such that $b_{I_{0}} \succ^{\lambda} a$, then weak IK-dominance of $b_{I_{0}}$ follows. Equivalently, the condition can be written as requiring that for all $q \in \mathcal{Q}_{R}, U\left(I_{0}, q\right)>U(I, q)$.

Now, recall that the function $(1-I)^{1-\gamma} A+I^{1-\gamma} B$, i strictly concave in $I$, with optimum at $I^{*}=\frac{1}{1+\left(\frac{A}{B}\right)^{1 / \gamma}}$. The optimum is thus strictly increasing in $B$, and where

$$
\begin{aligned}
A & =\sum_{n} \lambda^{n}\left(f_{0}^{n}\right)^{1-\gamma}, \\
B & =\sum_{n} \lambda^{n} \sum_{m}\left(f_{1 m}^{n}\right)^{1-\gamma} z_{m} q_{m}^{n},
\end{aligned}
$$

in the economy without taxes. Define

$$
C=\inf _{q \in \mathcal{Q}_{R}} \sum_{m}\left(f_{1 m}^{n}\right)^{1-\gamma} z_{m} q_{m}
$$

It then follows that $C>B$, because assume to the contrary that

$$
C=\sum_{m}\left(f_{1 m}^{n}\right)^{1-\gamma} z_{m} q_{m}=\sum_{n} \lambda^{n} \sum_{m}\left(f_{1 m}^{n}\right)^{1-\gamma} z_{m} q_{m} \leq B
$$

Since the unique optimal consumption share with homogeneous beliefs is $\hat{f}_{m}^{n}=\frac{\left(\lambda^{n}\right)^{1 / \gamma}}{\sum_{n}\left(\lambda^{n}\right)^{1 / \gamma}}$, this in turn implies that

$$
\hat{C}=\sum_{n} \lambda^{n} \sum_{m}\left(\hat{f}_{1 m}^{n}\right)^{1-\gamma} z_{m} q_{m}=\left(\sum_{n}\left(\lambda^{n}\right)^{1 / \gamma}\right)^{\gamma} \sum_{m} z_{m} q_{m}<B .
$$

(recall that $1-\gamma<0$, so we wish to minimize $C$ and, moreover, that $B$ has variable consumption weights across states because of heterogeneous beliefs, so it is not the case that $f_{1 m}^{n}=\hat{f}_{1 m}^{n}$ for all $m$ and $n$ ). But, this would imply 
the inequality

$$
\begin{aligned}
\hat{B} & =\sum_{n} \sum_{m} \hat{f}_{1 m}^{n} z_{m} q_{m}^{n}=\sum_{n} \sum_{m} \frac{\left(\lambda^{n}\right)^{1 / \gamma}}{\left(\sum_{n^{\prime}}(\lambda)^{1 / \gamma}\right)^{1-\gamma}} z_{m} q_{m}^{n} \\
& =\sum_{B \in P^{A}} z_{B} \mathbb{P}(B) \sum_{\omega_{m} \in B} \sum_{n} \frac{\left(\lambda^{n}\right)^{1 / \gamma}}{\left(\sum_{n^{\prime}}(\lambda)^{1 / \gamma}\right)^{1-\gamma}} \mathbb{P}^{n}\left(\omega_{m} \mid B\right) \\
& =\sum_{B \in P^{A}} z_{B} \mathbb{P}(B) \sum_{n} \frac{\left(\lambda^{n}\right)^{1 / \gamma}}{\left(\sum_{n^{\prime}}(\lambda)^{1 / \gamma}\right)^{1-\gamma}} \\
& =C \\
& <B
\end{aligned}
$$

where we used the fact that agents agree on aggregate risk, in turn implying that $B$ was based on suboptimal consumption shares, a contradiction, and thus it must be that $C>B$.

Now, since $C>B$, it follows that $\bar{I}^{*}=\arg \max _{\bar{I}} U(\bar{I}, q)$ (which does not depend on $q \in \mathcal{Q}_{R}$, since agents agree on aggregate risk), satisfies $\bar{I}^{*}>I(0)$. Moreover, since $U(\bar{I}, q)$ is strictly concave in $\bar{I}$, it must then be that $\left.\frac{d U}{d I}\right|_{\bar{I}=I(0)}>0$, which together with $\frac{d I}{d \tau}>0$ implies that $U(I(\tau), q)>U(I(0), q)$ for some $\tau>0$.

Thus, the outcome with $\operatorname{tax} \tau$ IK-dominates the original equilibrium outcome with respect to Pareto weights $\lambda$, and therefore weakly IK-dominates that outcome. We are done.

\section{Proof of Proposition 13}

Production economy: In the production economy without aggregate uncertainty we have that $R_{m}=R$ for all $m=1, \ldots, M$. Hence, the unanimously reasonable value of the risk free rate is $R$. In equilibrium, the risk free rate is

$$
R_{f}=\left(\frac{R^{1-\gamma}}{R^{-\gamma}}\right)\left(\frac{\sum_{n=1}^{N} T_{m}^{\gamma}}{\sum_{n=1}^{N} T_{m}^{\gamma}}\right)=R
$$

Since the equilibrium risk free rate is always equal to the unanimously reasonable value, the risk free rate is thus not distorted.

Pure exchange economy: Let aggregate consumption at time zero be $C_{0}$ and the aggregate consumption at time 1 be $C_{1}$. Then, the unanimously reasonable value of the risk free rate is

$$
R_{f}=E\left[\left(\frac{C_{1}}{C_{0}}\right)^{-\gamma} \mid q\right]=\left(\frac{C_{1}}{C_{0}}\right)^{-\gamma},
$$

for all $q \in \mathcal{Q}_{R}$. In equilibrium the risk free rate is

$$
\begin{aligned}
R_{f} & =E\left[\left(\frac{\tilde{c}_{1}^{n}}{c_{0}^{n}}\right)^{-\gamma} \mid q^{n}\right] \\
& =\left(\frac{C_{1}}{C_{0}}\right)^{-\gamma}\left(\frac{\sum_{m=1}^{M} T_{m}^{\gamma}}{L^{\gamma}}\right) .
\end{aligned}
$$

Since $\left(\frac{\sum_{m=1}^{M} T_{m}^{\gamma}}{L^{\gamma}}\right) \neq 1$ for $\gamma \neq 1$ due to Jensen's inequality, the risk free rate is always distorted.

\section{Proof of Proposition 14:}

The result is an immediate consequence of absence of arbitrage, since any payoff that is in the span of the production technologies can be replicated. As the production technologies themselves must be correctly priced in equilibrium, any asset in the span of the production technologies will also be correctly priced. 


\section{References}

Baker, S., B. Hollifield, and E. Osambela. 2014. Disagreement, Speculation, and Aggregate Investment. Working Paper, Carnegie Mellon University,.

Basak, S. 2005. Asset pricing with heterogeneous beliefs. Journal of Banking and Finance 29:2849?2881.

Blume, L. E., T. Cogley, D. A. Easley, T. J. Sargent, and V. Tsyrennikov. 2014. The Case for Incomplete Markets. Working paper, Cornell University.

Blundell, R., M. Browning, and C. Meghir. 1994. Consumer Demand and the Life-Cycle Allocation of Household Expenditures. Review of Economic Studies 61:57-80.

Brunnermeier, M. K., A. Simsek, and W. Xiong. 2013. A Welfare Criterion for Models with Distorted Beliefs. Quarterly Journal of Economics, forthcoming.

Dávila, E. 2014. Optimal Financial Transaction Taxes. Working Paper, New York University,.

Ehling, P., M. Gallmeyer, C. Heyerdahl-Larsen, and P. Illeditsch. 2013. Disagreement about inflation and the yield curve. Working Paper.

Fedyk, Y., C. Heyerdahl-Larsen, and J. Walden. 2013. Market Selection in a Multi-Asset Economy. Review of Finance $17: 1179-1237$.

Fleurbaey, M. 2010. A defense of the ex-post evaluation of risk. Working paper, University of Paris Descartes.

Gayer, G., I. Gilboa, L. Samuelson, and D. Schmeidler. 2013. Pareto Efficiency with Different Beliefs. Working paper, Tel Aviv University.

Gilboa, I., L. Samuelson, and D. Schmeidler. 2013. No-Betting-Pareto Dominance. Econometrica, forthcoming.

Gilchrist, S., C. P. Himmelberg, and G. Huberman. 2005. Do stock price bubbles influence corporate investment. Journal of Monetary Economics 52:805-827.

Hammond, P. 1981. Ex-Ante and Ex-Post Welfare Optimality under Uncertainty. Economica 48:235-250.

Harris, R. 1978. Ex-Post Efficiency and Resource Allocation under Uncertainty. Review of Economic Studies 45:427436.

Harris, R., and N. Olewiler. 1979. The welfare economics of ex post optimality. Economica 46:137-147.

Hausman, D., and M. McPherson. 1994. Preference, Belief, and Welfare. American Economic Review, Papers 65 Proceedings 84:396-400.

Havranek, T., R. Horvath, and Z. Irsova. 2013. Cross-Country Heterogeneity in Intertemporal Substitution. Working Paper, Charles University, Prague.

Hicks, J. R. 1946. Value and Capital. 2nd ed. Oxford University Press.

Higham, N. J., and S. H. Cheng. 1998. Modifying the inertia of matrices arising in optimization. Linear Algebra and its Applications 275-276:261-279.

Hirshleifer, J. 1975. Speculation and Equilibrium: Information, Risk and Markets. Quarterly Journal of Economics 89:519-542.

Keynes, J. M. 1930. A Treatise on Money. Harcourt, Brace \& Company.

Kubler, F., and K. Schmedders. 2012. Financial Innovation and Asset Price Volatility. American Economic Review, Papers and Proceedings 102:147-51.

Mongin, M. 1995. Consistent Bayesian Aggregation. Journal of Economic Theory 66:313-351.

Pastor, L., and P. Veronesi. 2006. Was there a Nasdaq bubble in the late 1990s. Journal of Financial Economics 81:61-100.

Pollak, R. A. 1998. Imagined Risk and Cost-Benefit Analysis. American Economic Review, Papers 85 Proceedings $88: 376-380$.

Portney, P. R. 1992. Trouble in Happyville. Journal of Policy Analysis and Management 11:131-132. 
Salanie, F., and N. Treich. 2009. Regulation in Happyville. Economic Journal 119:665-679.

Savage, L. J. 1954. The Foundations of Statistics. Wiley.

Scheinkman, J., and W. Xiong. 2003. Overconfidence and speculative bubbles. Journal of Political Economy 111:11831219.

Shiller, R. J. 2000. Irrational Exuberance. Princeton University Press.

Simsek, A. 2013. Speculation and Risk Sharing with New Financial Assets. Quarterly Journal of Economics, forthcoming .

Starr, R. M. 1973. Optimal Production and Allocation under Uncertainty. Quarterly Journal of Economics 87:81-95.

Vissing-Jorgensen, A. 2002. Limited Asset Market Participation and the Elasticity of Intertemporal Substitution. Journal of Political Economy 110:925-853.

Working, H. 1953. Futures Trading and Hedging. American Economic Review 43:314-43.

Yan, H. 2008. Natural selection in financial markets: Does it work? Management Science 54:1935-1950. 